\title{
Semiclassical aspects of (chaotic) quantum systems
}

Part 1: Fundamental ideas, $S$-matrix-based observables

Klaus Richter

University of Regensburg, Germany 


\section{Semiclassical aspects of}

\section{(chaotic) quantum systems}




\section{Semiclassical aspects of}

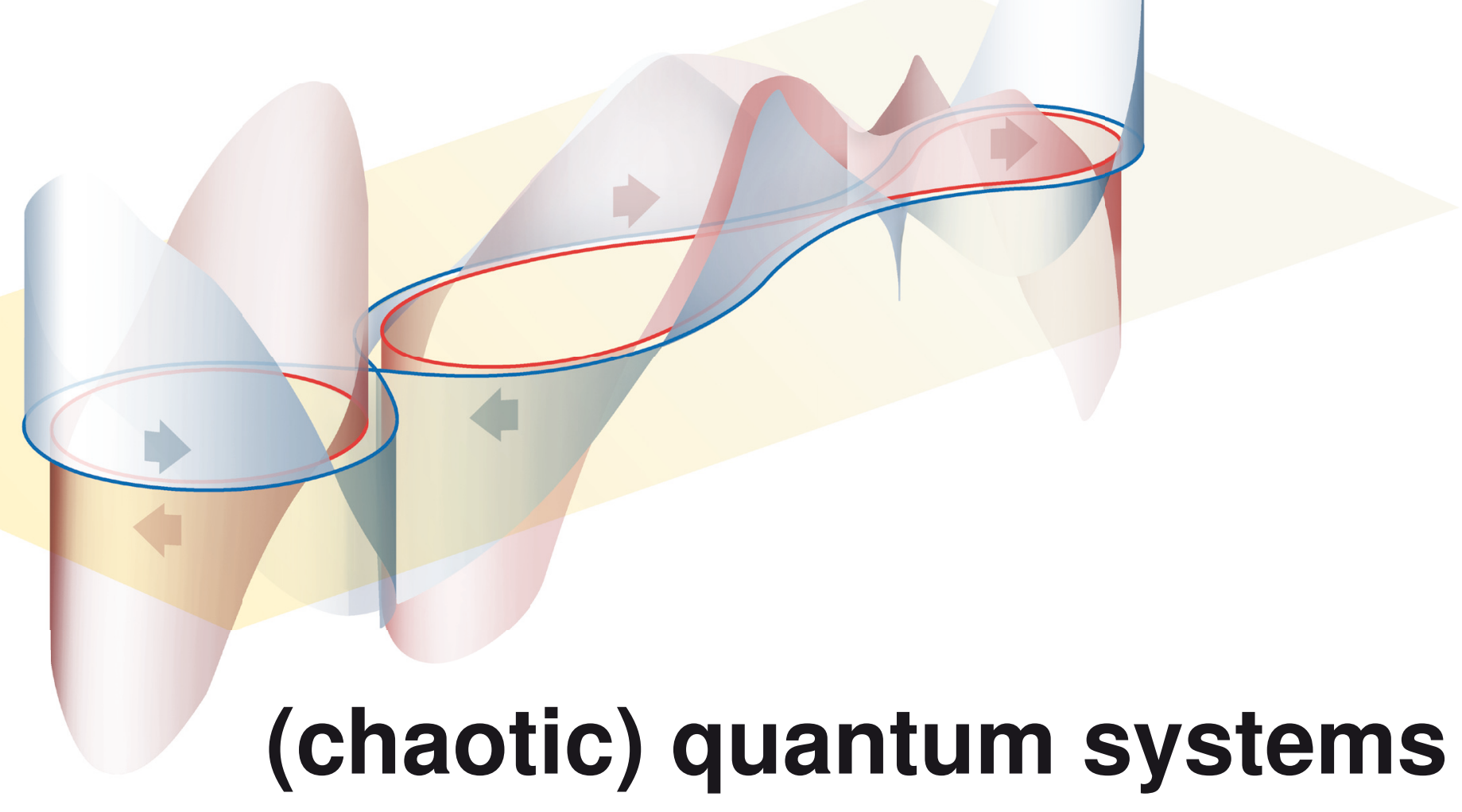

Semiclassics 1: fundamental ideas,

$S$-matrix-based observables

(Klaus Richter, University of Regensburg) 


\section{Semiclassical aspects of}

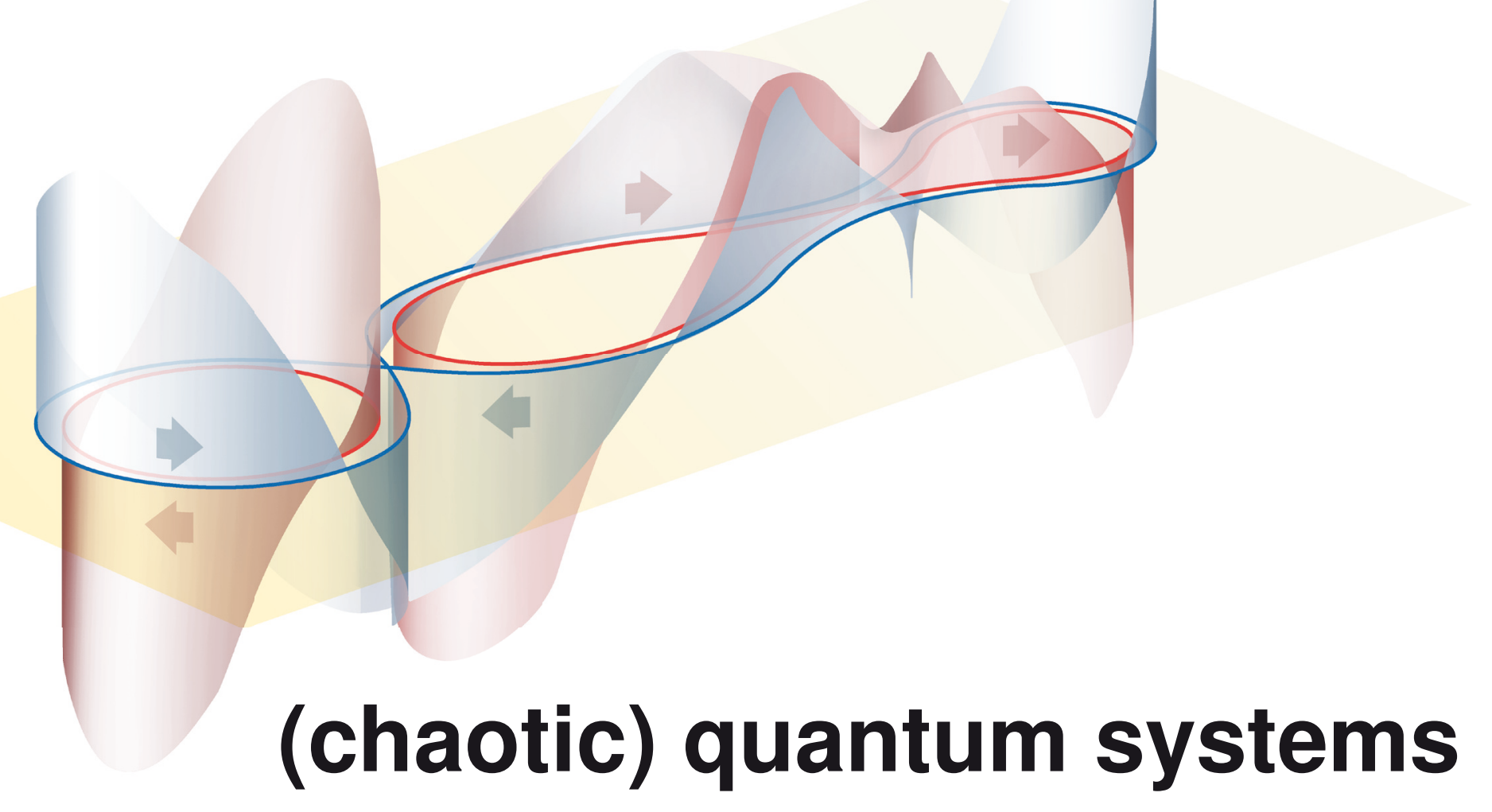

Semiclassics 2: theory of spectral statistics (Sebastian Müller) 


\title{
Universality in chaotic quantum systems
}

\author{
Random Matrix Theory (RMT)
} $\downarrow$

classical

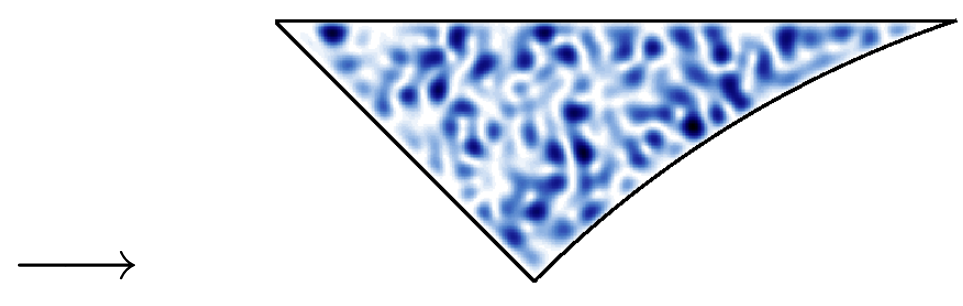

$\longrightarrow$ quantum

Advanced Semiclassical Theory 


\title{
Universality in chaotic quantum systems
}

\author{
Random Matrix Theory (RMT)
}

classical

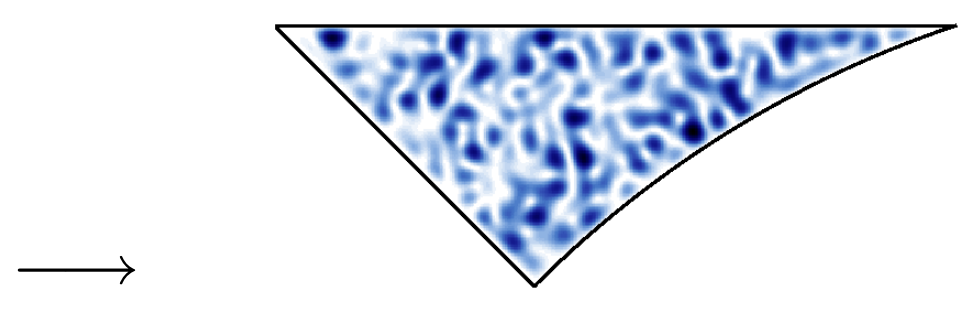

$\longrightarrow$ quantum

\author{
Advanced Semiclassical Theory \\ key: classical orbit correlations
}




\section{Correlations in classical dynamics}




\section{Integrable systems}

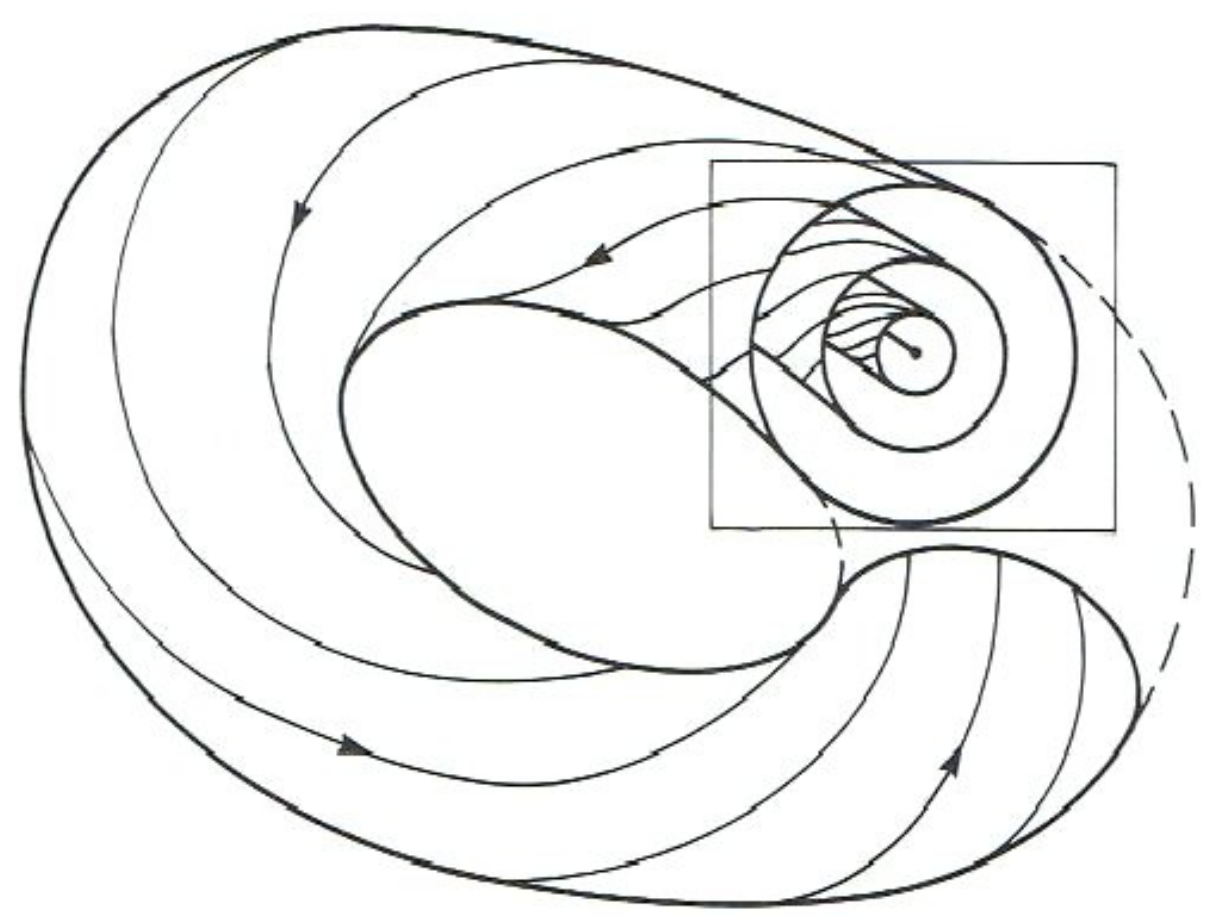

$\Rightarrow$ dynamics organized on tori in phase space

$\Rightarrow$ families of orbits with the same length and classical action $S=\oint \mathbf{p} d \mathbf{q}$ 


\section{Chaotic systems}

Example: Chaotic billiard:
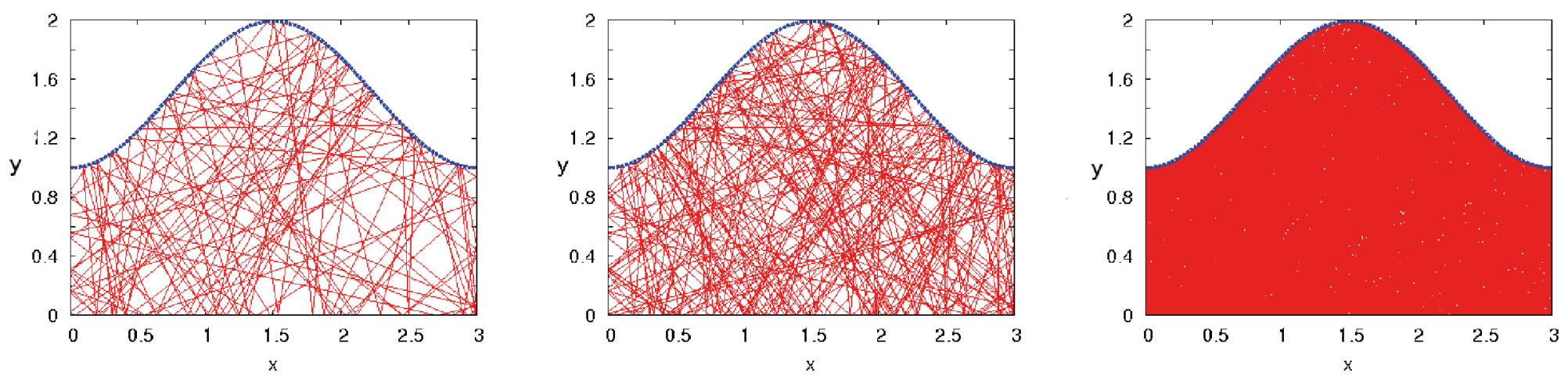

Poincaré surface of section:
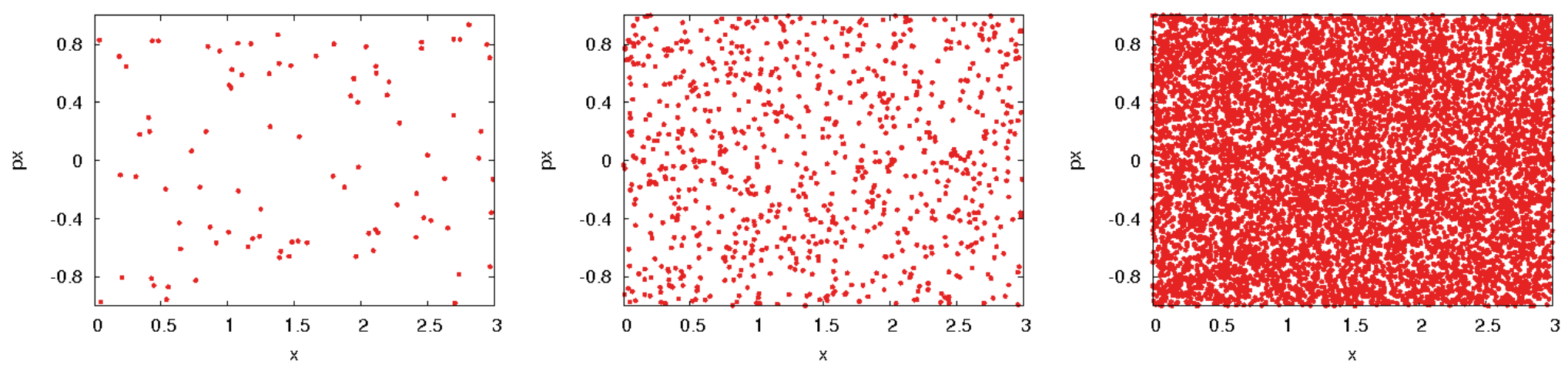

$\Rightarrow$ ergodic dynamics 


\section{Chaotic systems}

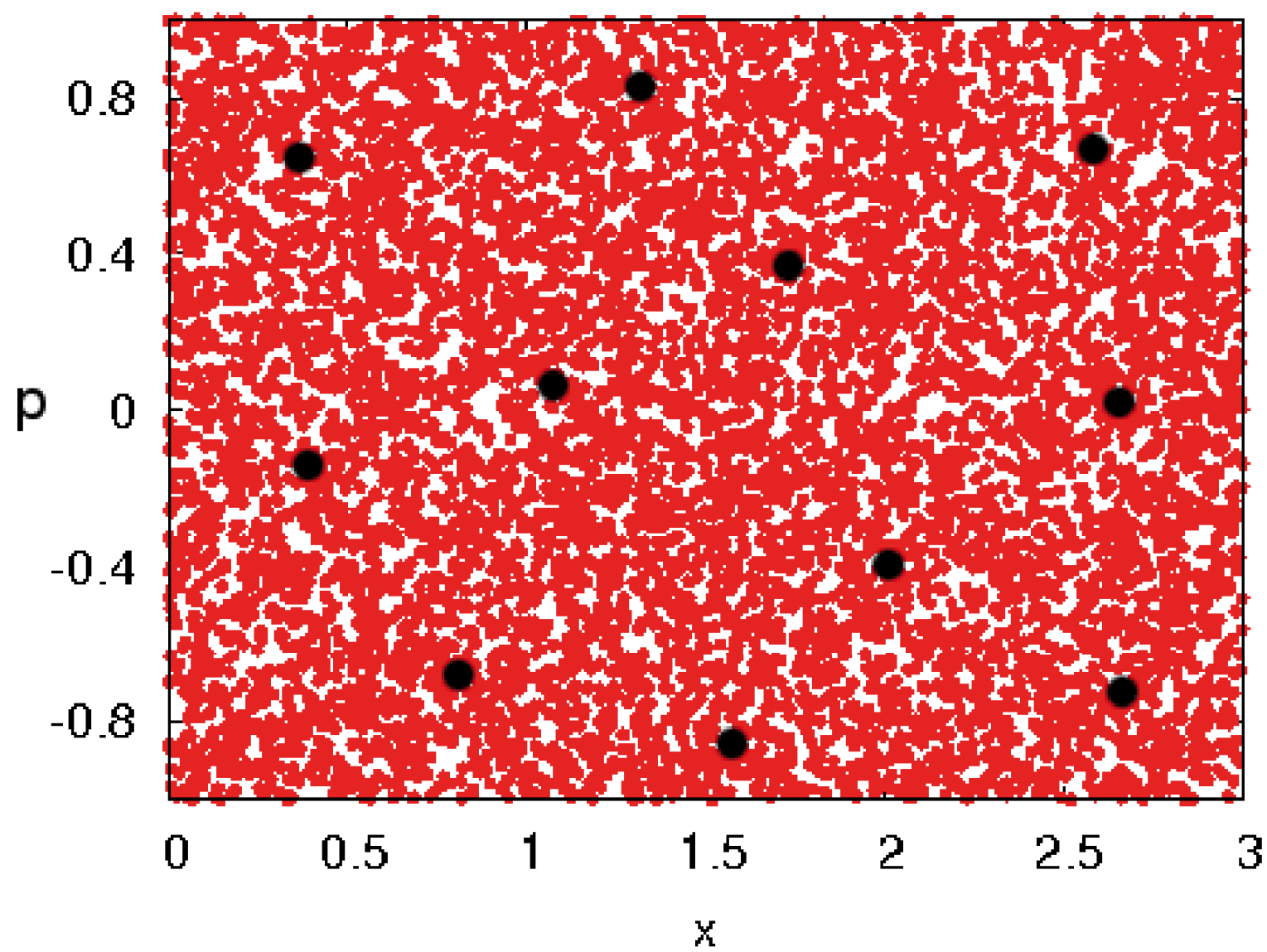

H. Poincaré (1892):

- periodic orbits are densely distributed; $\quad \# \mathrm{po} \sim e^{\mu T}$

- periodic orbits provide clue to overall behavior 


\section{Chaotic systems}

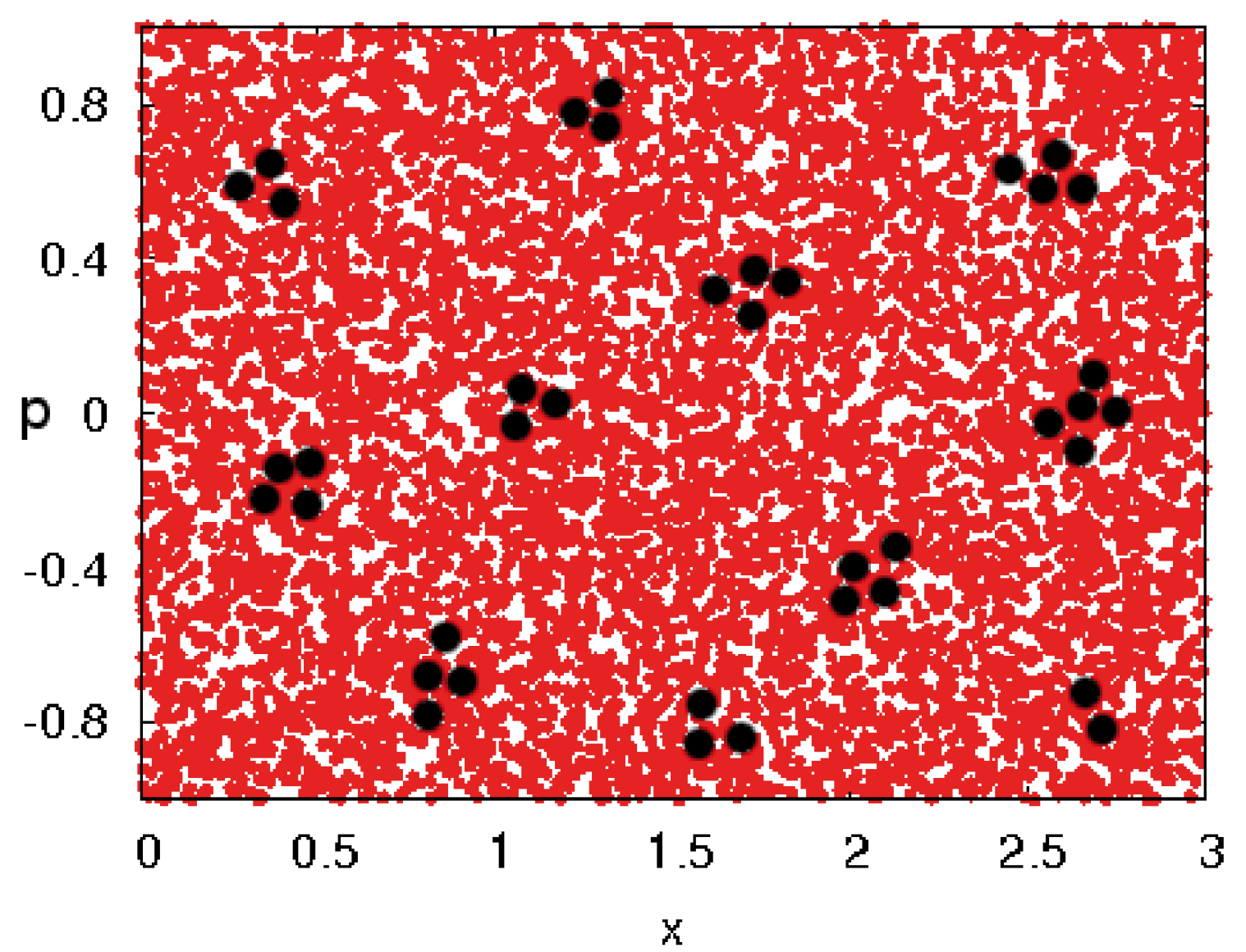

$\Rightarrow$ subtle correlations between periodic orbits

$\Rightarrow$ periodic orbits appear in multiplets ! 


\section{periodic orbit doublets}

Example: correlated periodic orbit pair in the hyperbola billiard:
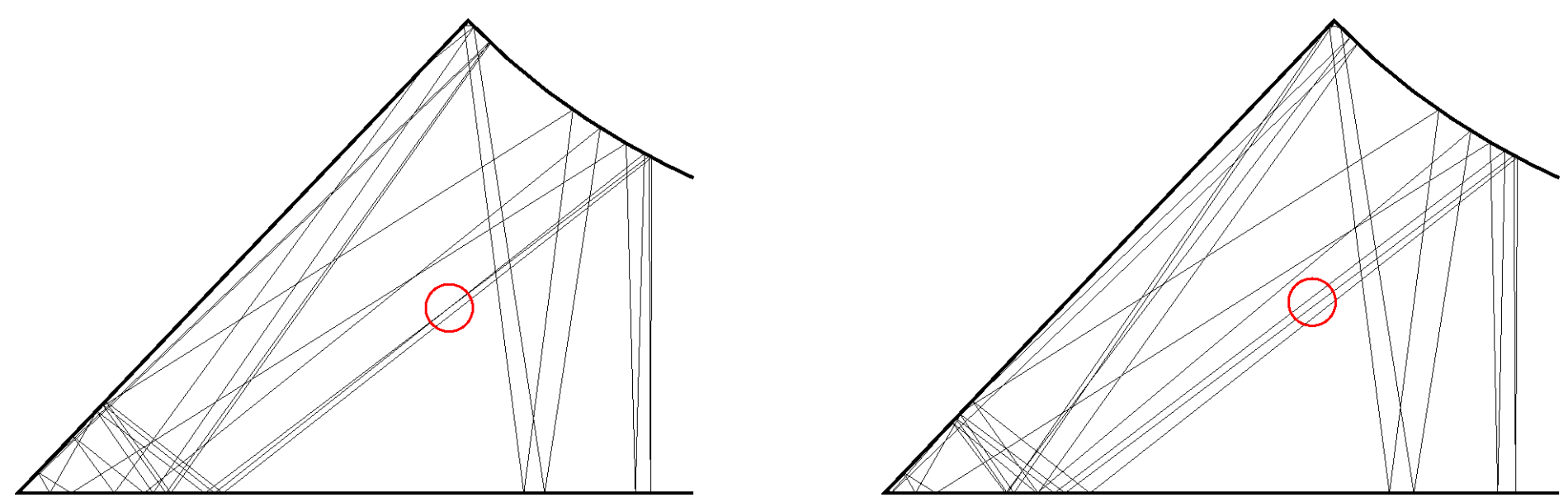

M. Sieber

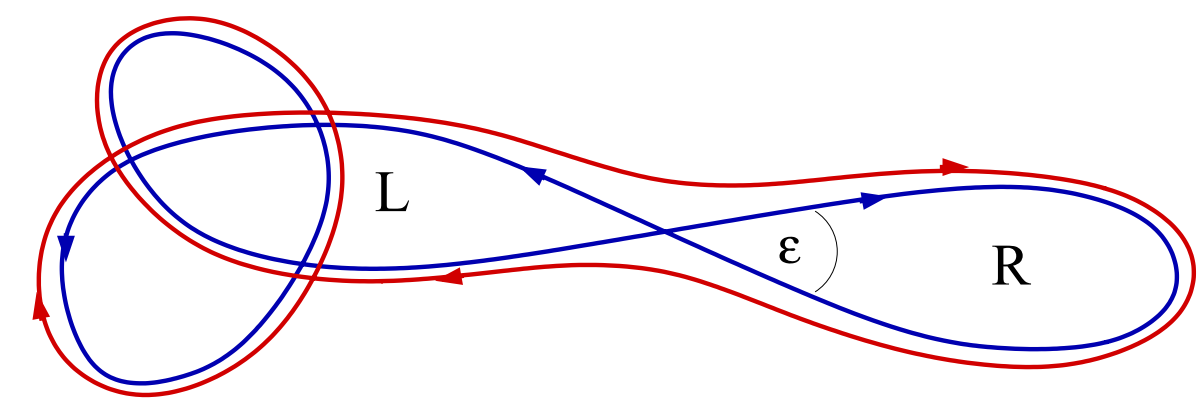

$\Rightarrow$ action difference $S_{1}-S_{2} \sim \epsilon^{2} p^{2} \rightarrow 0$

M. Sieber and KR (2001) 


\section{periodic orbit doublets}

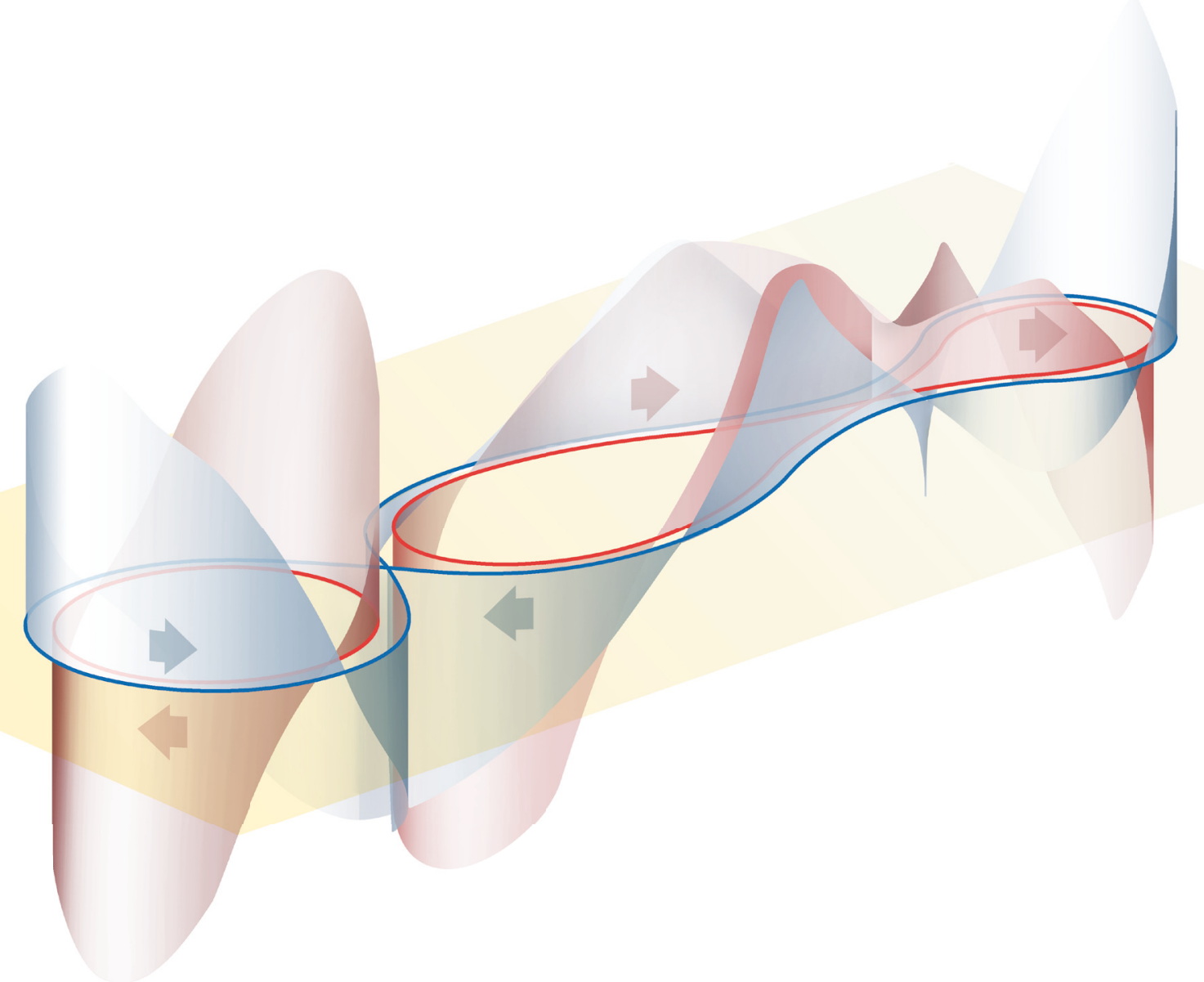




\section{periodic orbit structures}

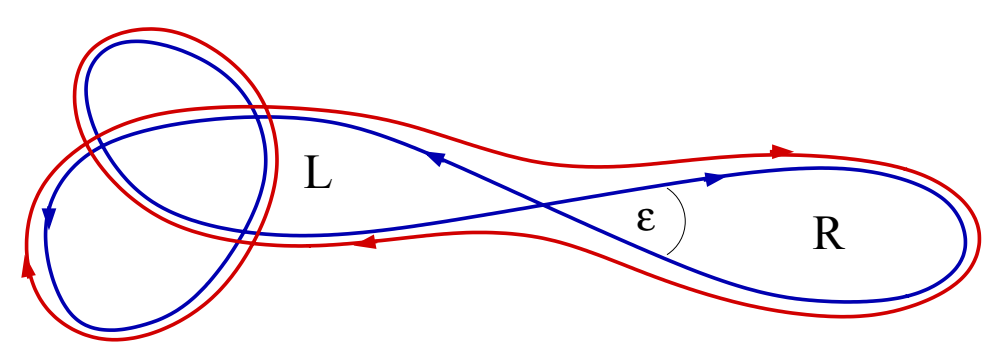

po pairs

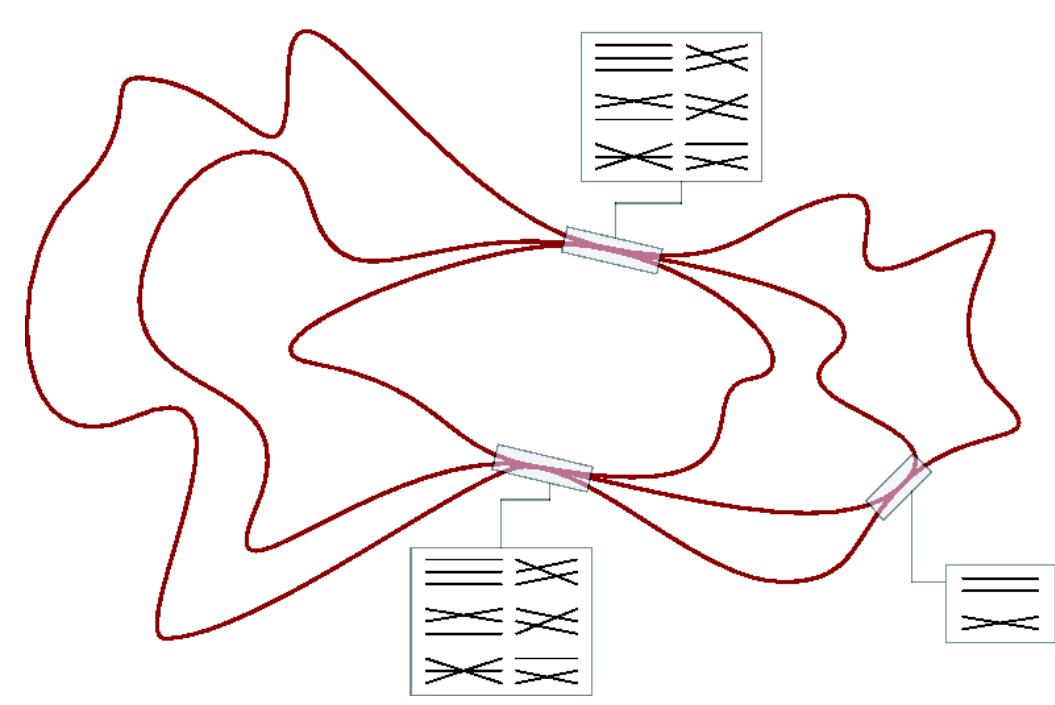

po bundels Müller et al. (2009) 


\section{periodic orbit structures}

periodic orbit webs

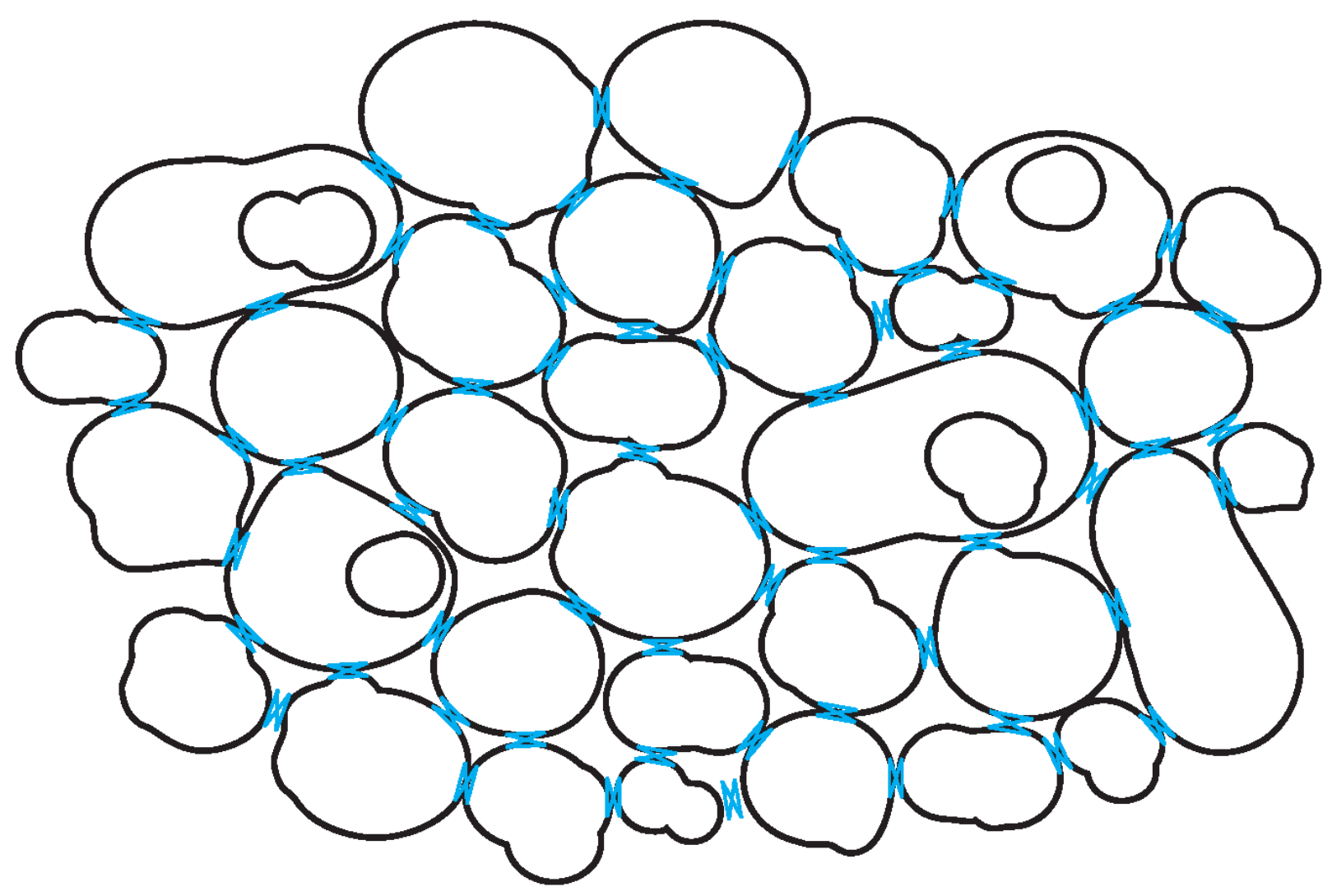

$\Rightarrow$ role in classical mechanics ?

$\Rightarrow$ semiclassical backbone of chaotic quantum systems ! 


\section{Correlations in spectral statistics}




\section{Quantum dynamics at mesoscopic scales}

wavepacket evolution in a chaotic confinment:
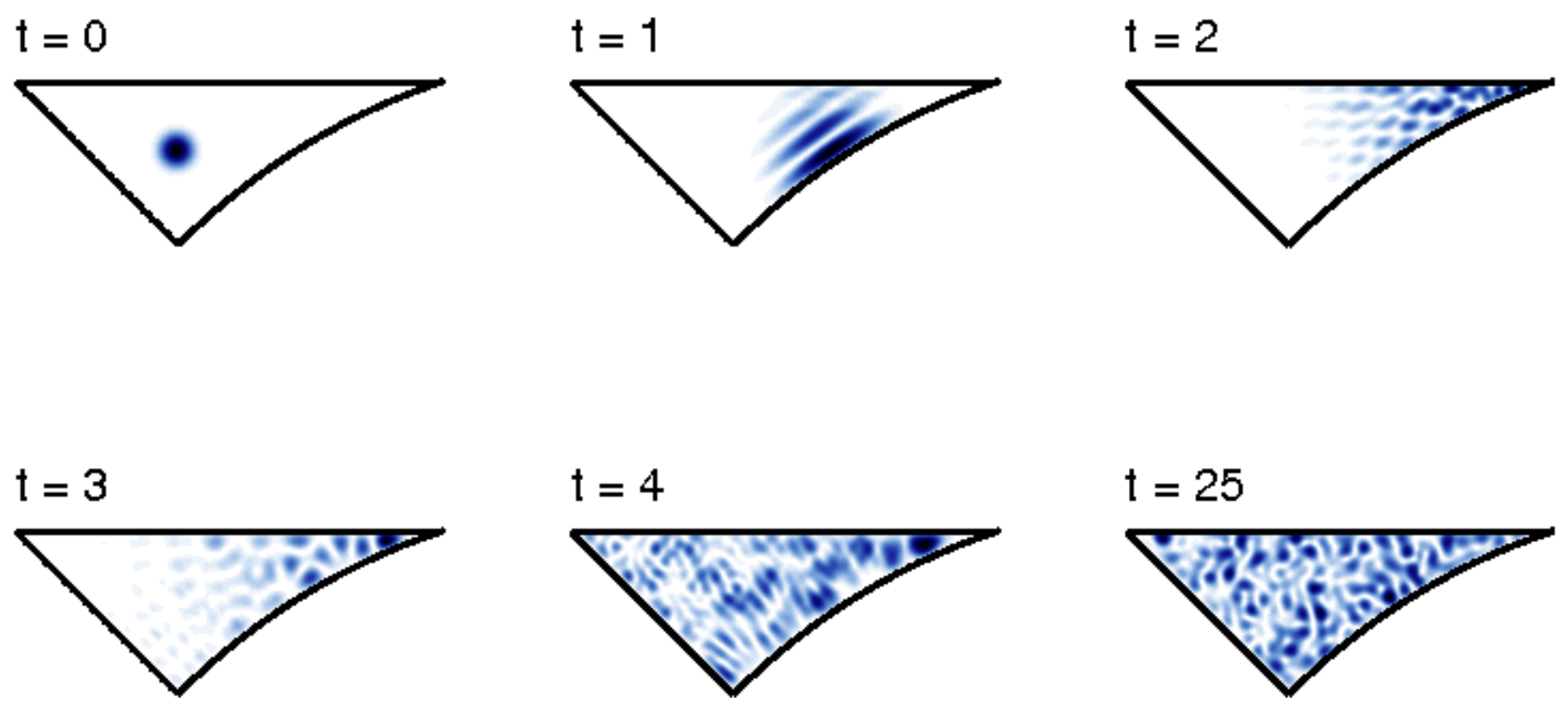

(A. Goussev)

- complex wavefunction morphology

- semiclassical regime: $\lambda_{\mathrm{dB}} \ll$ system size $\mathcal{L}$ 


\section{Universality in quantum spectra}

\section{Typical level sequences (Bohigas et al. (1983))}

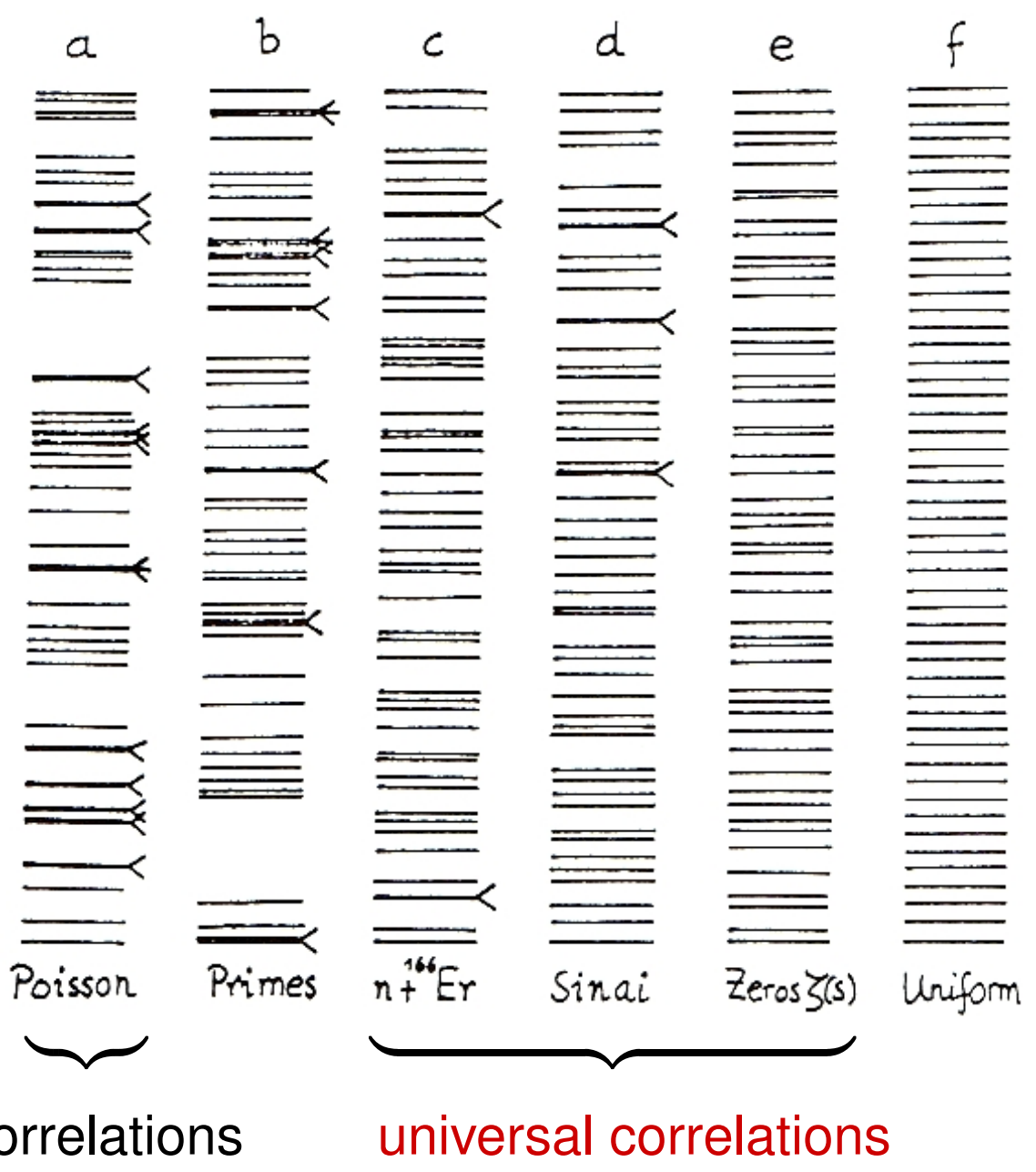

no correlations universal correlations 


\section{Universality in quantum spectra}

Distribution of level spacings following from Random Matrix Theory (RMT)

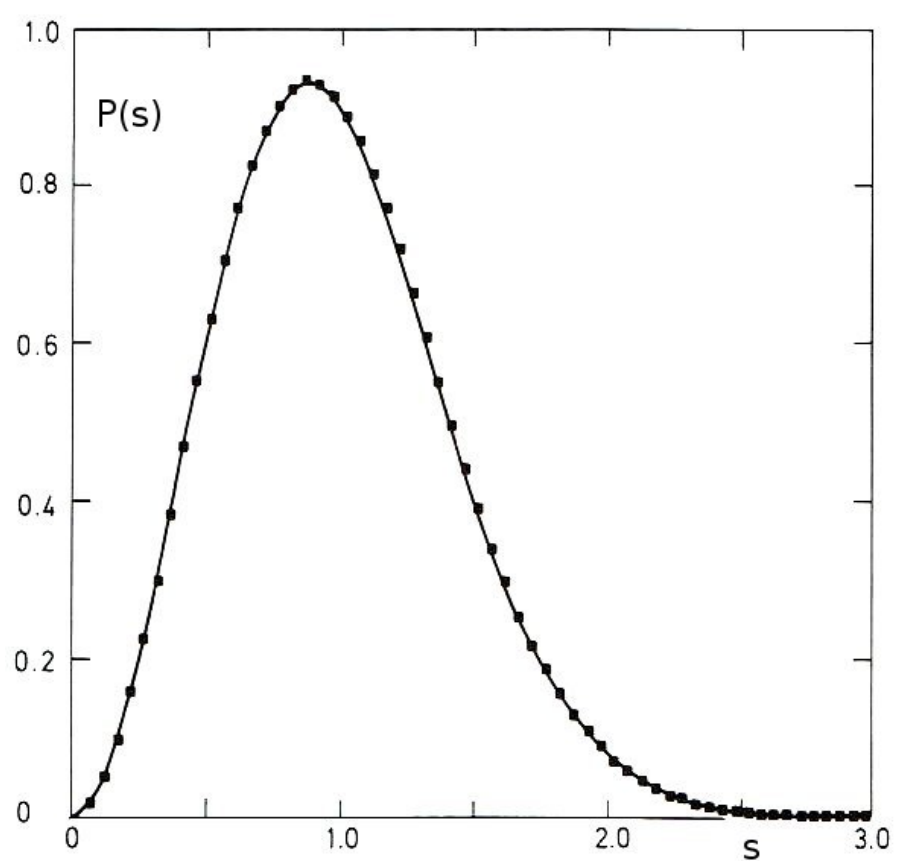

Odlyzko (1989)

$\Rightarrow$ RMT predicts universality of level fluctuations: for interacting systems, Riemann zeros, classically chaotic systems

Bohigas, Giannoni, Schmit (1983) 


\section{Universality in chaotic quantum systems}

\section{Random Matrix Theory (RMT)}

classical

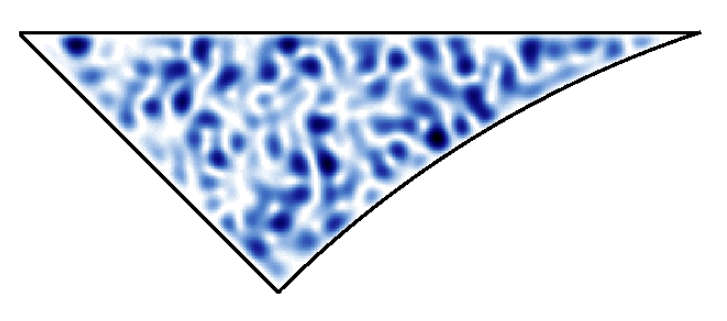

\section{Advanced Semiclassical Theory}

classical orbit correlations $\Rightarrow$ RMT predictions

$\Rightarrow$ go beyond RMT ! 


\section{Spectral form factor}

- Quantum density of states: $d(E)=\sum_{n} \delta\left(E-E_{n}\right)=\bar{d}(E)+d_{\mathrm{osc}}(E)$ 


\section{Spectral form factor}

- Quantum density of states: $d(E)=\sum_{n} \delta\left(E-E_{n}\right)=\bar{d}(E)+d_{\text {osc }}(E)$

- Spectral form factor:

$$
K(t) \sim \int_{-\infty}^{\infty} \mathrm{d} \eta\left\langle d_{\mathrm{osc}}\left(E+\frac{\eta}{2}\right) d_{\mathrm{osc}}\left(E-\frac{\eta}{2}\right)\right\rangle_{E} \exp (2 \pi i \eta t)
$$

- ||||||||||||||||||||||||||||||||||||||||||||||

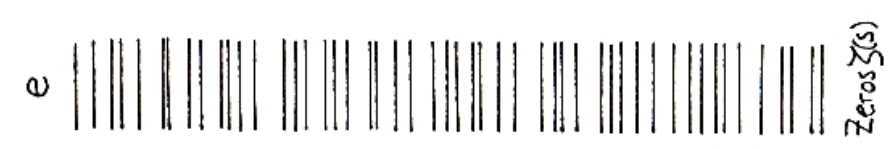

• ||| || || || ||||||||||||||||||||||||||||||||||:

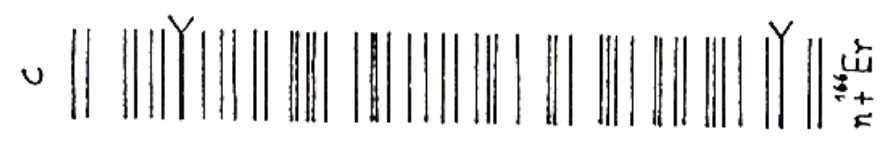

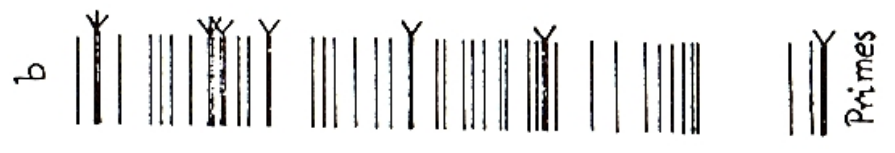

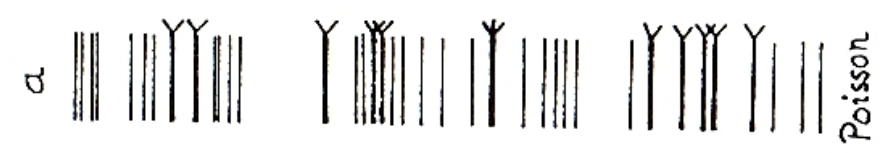




\section{Spectral form factor}

- Quantum density of states: $d(E)=\sum_{n} \delta\left(E-E_{n}\right)=\bar{d}(E)+d_{\mathrm{osc}}(E)$

- Spectral form factor:

$$
K(t) \sim \int_{-\infty}^{\infty} \mathrm{d} \eta\left\langle d_{\mathrm{osc}}\left(E+\frac{\eta}{2}\right) d_{\mathrm{osc}}\left(E-\frac{\eta}{2}\right)\right\rangle_{E} \exp (2 \pi i \eta t)
$$

$$
\tau=T / T_{\mathrm{H}} \quad ; \quad T_{\mathrm{H}}=2 \pi \hbar \bar{d}(E)
$$

chaotic system with time reversal symmetry (quartic oscillator)

RMT form factor of the Gaussian Orthogonal Ensemble (GOE)

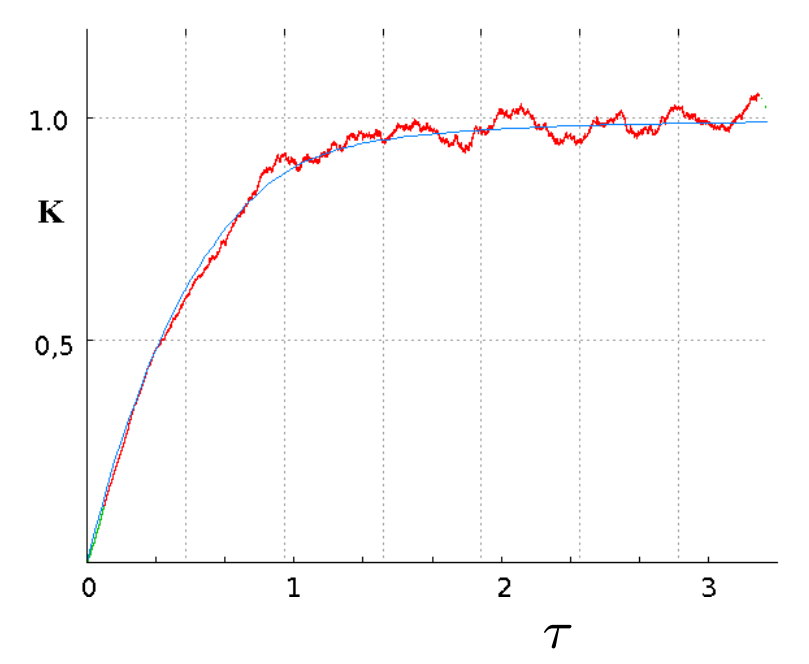

$$
K^{\mathrm{GOE}}(\tau)=2 \tau-\tau \log (1+2 \tau)=2 \tau-2 \tau^{2}+\ldots \quad \text { for } \quad \tau<1
$$




\section{Form factor: Semiclassical evaluation}

- Gutzwiller trace formula: coherent sum over periodic orbits

$$
d_{\mathrm{osc}}(E)=\frac{1}{\pi \hbar} \operatorname{Re} \sum_{\gamma} A_{\gamma} e^{i S_{\gamma}(E) / \hbar} \quad ; \quad S_{\gamma}(E) \gg \hbar
$$




\section{Form factor: Semiclassical evaluation}

- Gutzwiller trace formula: coherent sum over periodic orbits

$$
d_{\mathrm{osc}}(E)=\frac{1}{\pi \hbar} \operatorname{Re} \sum_{\gamma} A_{\gamma} e^{i S_{\gamma}(E) / \hbar} \quad ; \quad S_{\gamma}(E) \gg \hbar
$$

- Semiclassical representation of the form factor:

$$
K(\tau) \sim \sum_{\gamma, \gamma^{\prime}}\left\langle A_{\gamma} A_{\gamma^{\prime}}^{*} e^{i\left(S_{\gamma}-S_{\gamma^{\prime}}\right) / \hbar} \delta\left(T-\frac{T_{\gamma}+T_{\gamma^{\prime}}}{2}\right)\right\rangle_{E}
$$

- non-vanishing contributions from pairs of correlated orbits

- diagonal approximation: $\gamma=\gamma^{\prime}$ (Berry (1985))

$$
K(\tau) \simeq \frac{2}{T_{\mathrm{H}}} \sum_{\gamma}\left|A_{\gamma}\right|^{2} \delta\left(T-T_{\gamma}\right)
$$

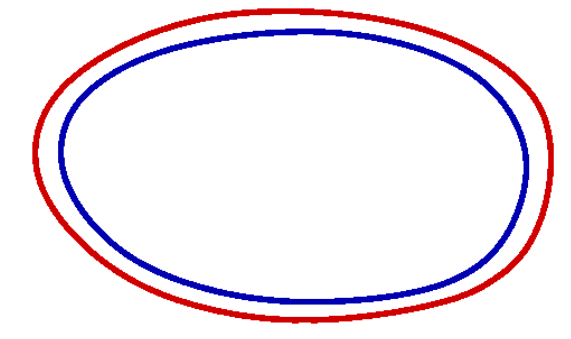

- Sum rule (Hannay, Ozorio de Almeida (1985))

$$
\sum_{\gamma}\left|A_{\gamma}\right|^{2} \delta\left(T-T_{\gamma}\right) \simeq T \quad \Longrightarrow \quad K(\tau) \approx 2 \tau
$$




\section{Form factor: Off-diagonal contributions}

- leading-order loop contribution:

- action difference:

$$
\Delta S(\varepsilon) \simeq p^{2} \varepsilon^{2} /(2 m \lambda)
$$

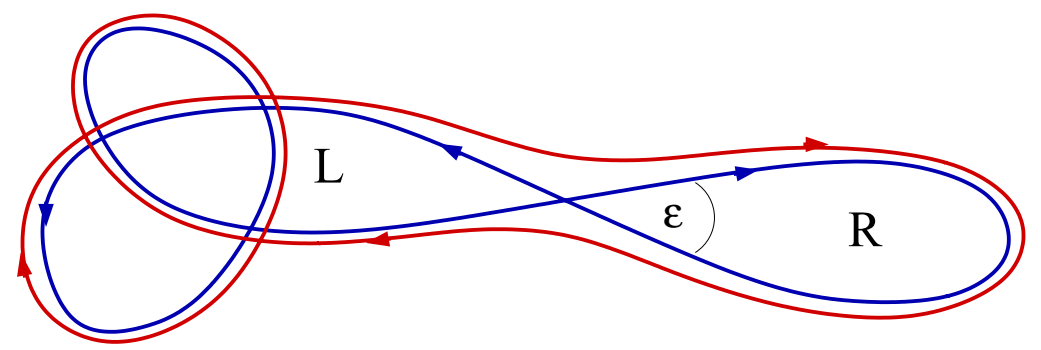

- contribution to form factor:

$$
K_{\mathrm{off}}(\tau) \sim \operatorname{Re} \int_{0}^{\pi} \mathrm{d} \varepsilon \sum_{\gamma}\left|A_{\gamma}\right|^{2} e^{i \Delta S(\varepsilon) / \hbar} \delta\left(T-T_{\gamma}\right) P(\varepsilon, T)
$$

- density of crossings $P(\varepsilon, T)$ ? 


\section{Crossing distribution}

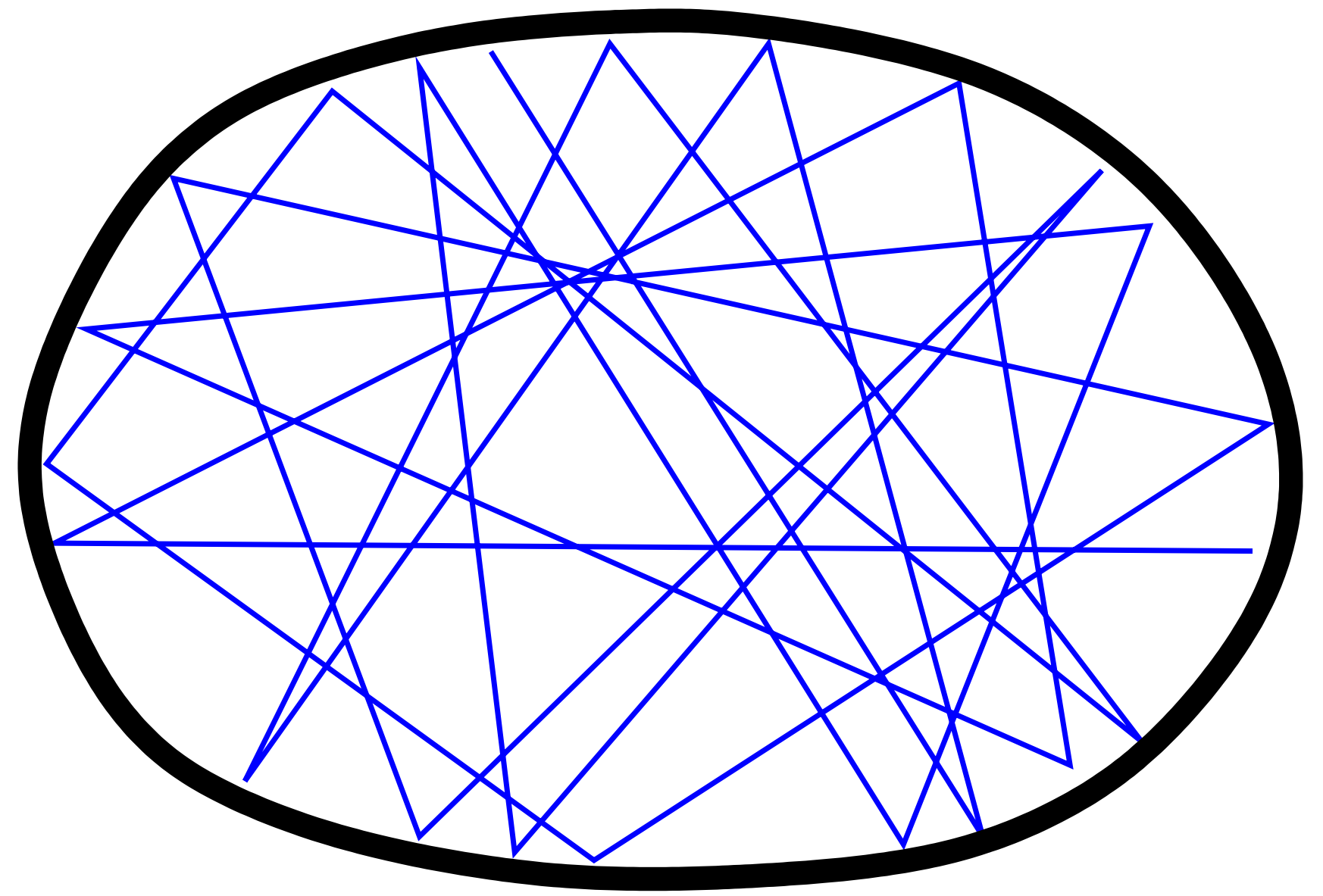

$$
P(\varepsilon, T) ?
$$




\section{Crossing distribution}

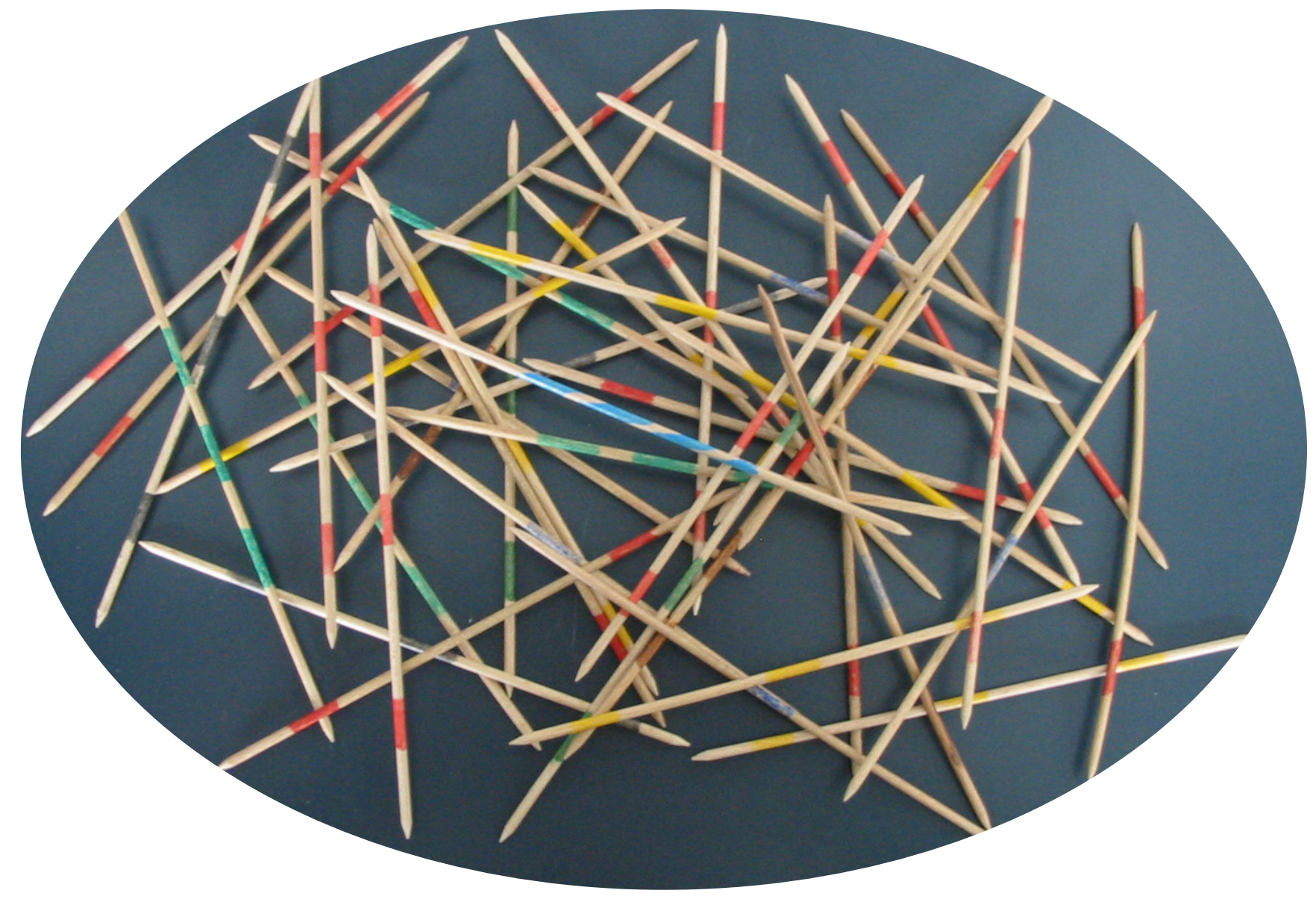

$$
T=N T_{0} \quad ; \quad P(\varepsilon, T) \sim N^{2} \sim T^{2}
$$




\section{Crossing distribution}

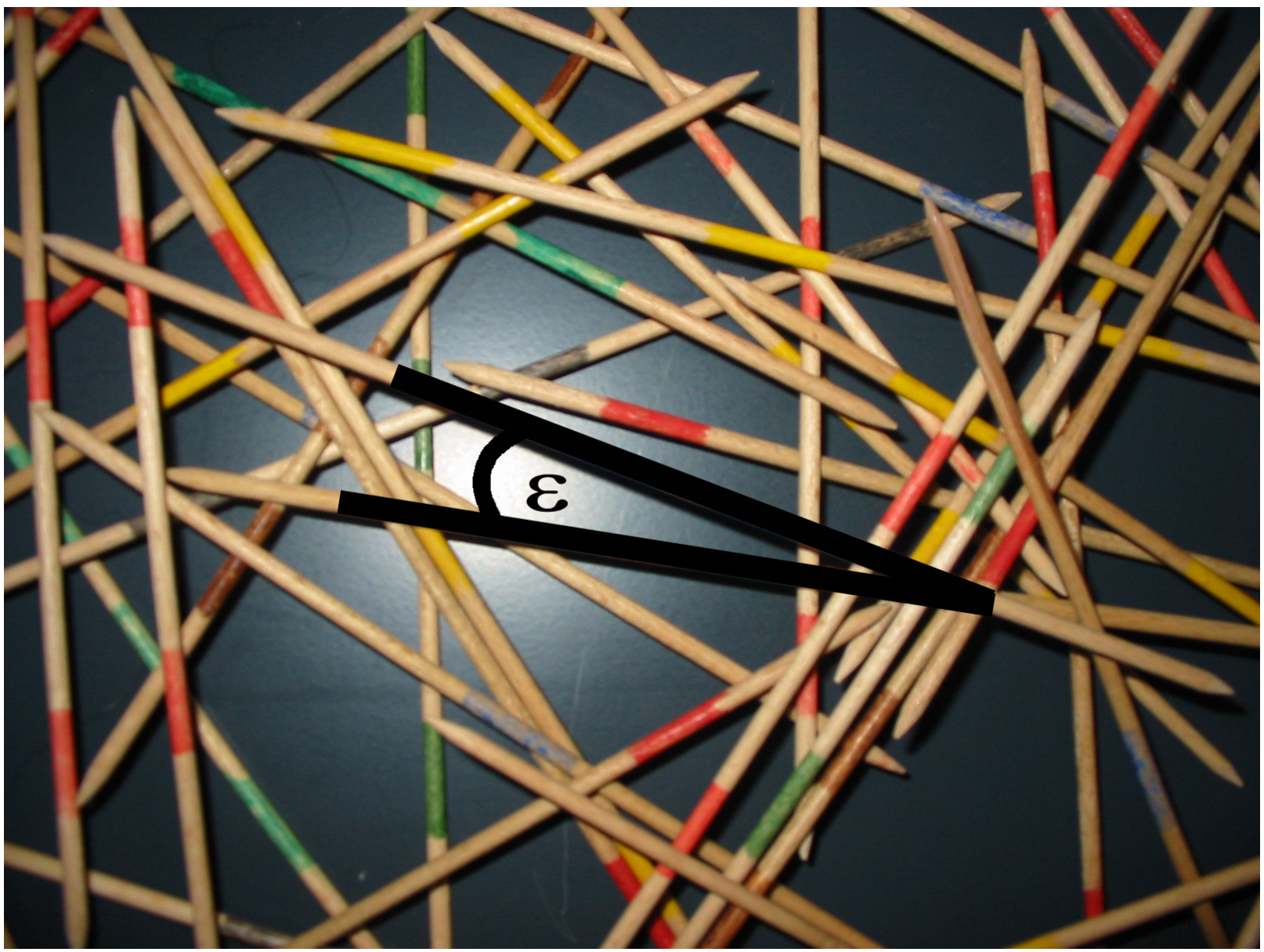

$$
P(\varepsilon, T) \sim \sin \varepsilon T^{2}
$$




\section{Crossing distribution}

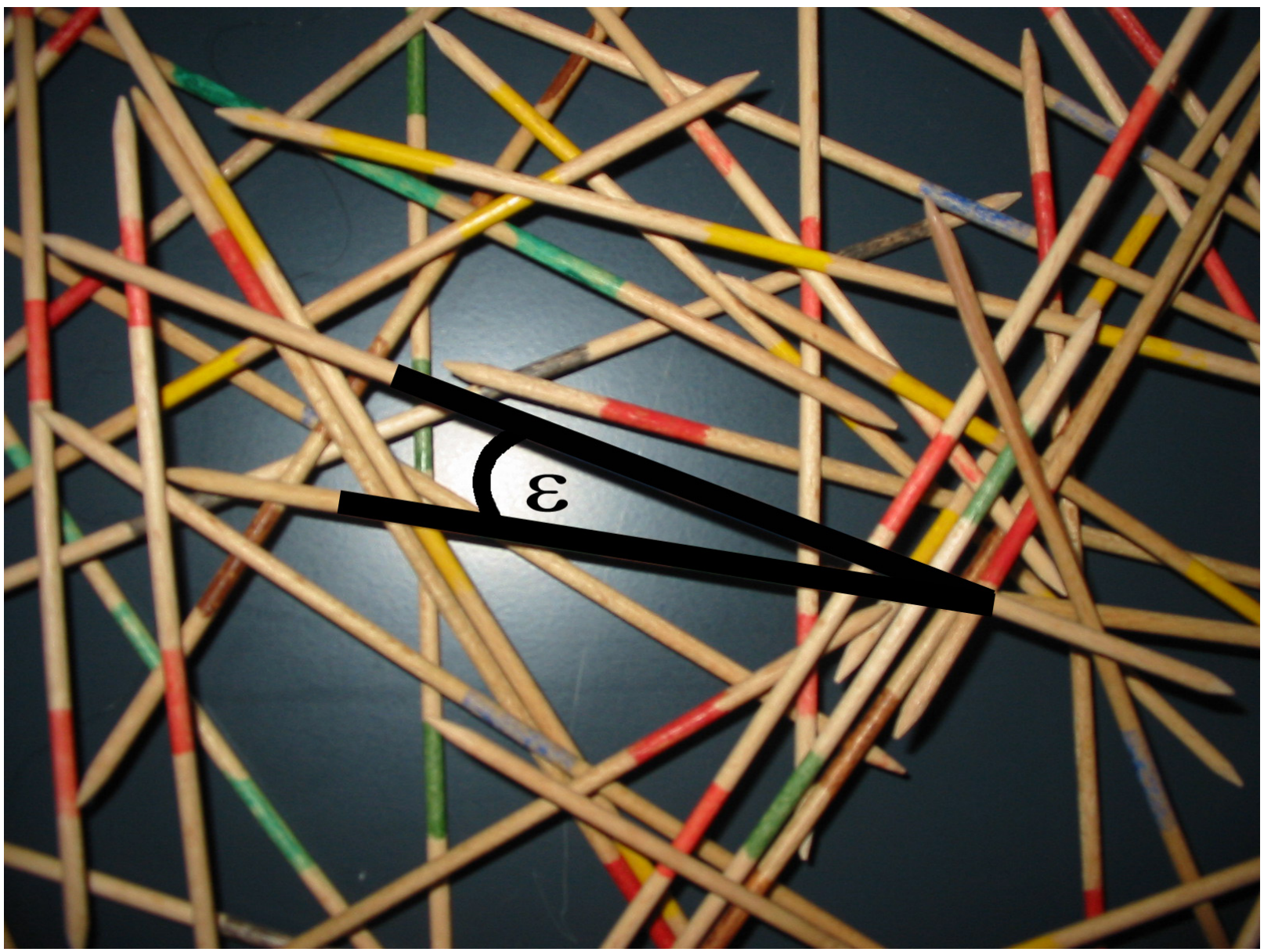

but: minimal loop time:

$$
c=\epsilon e^{\lambda T_{\mathrm{enc}}(\varepsilon) / 2} \quad ; \quad c \sim \pi
$$




\section{Crossing distribution}

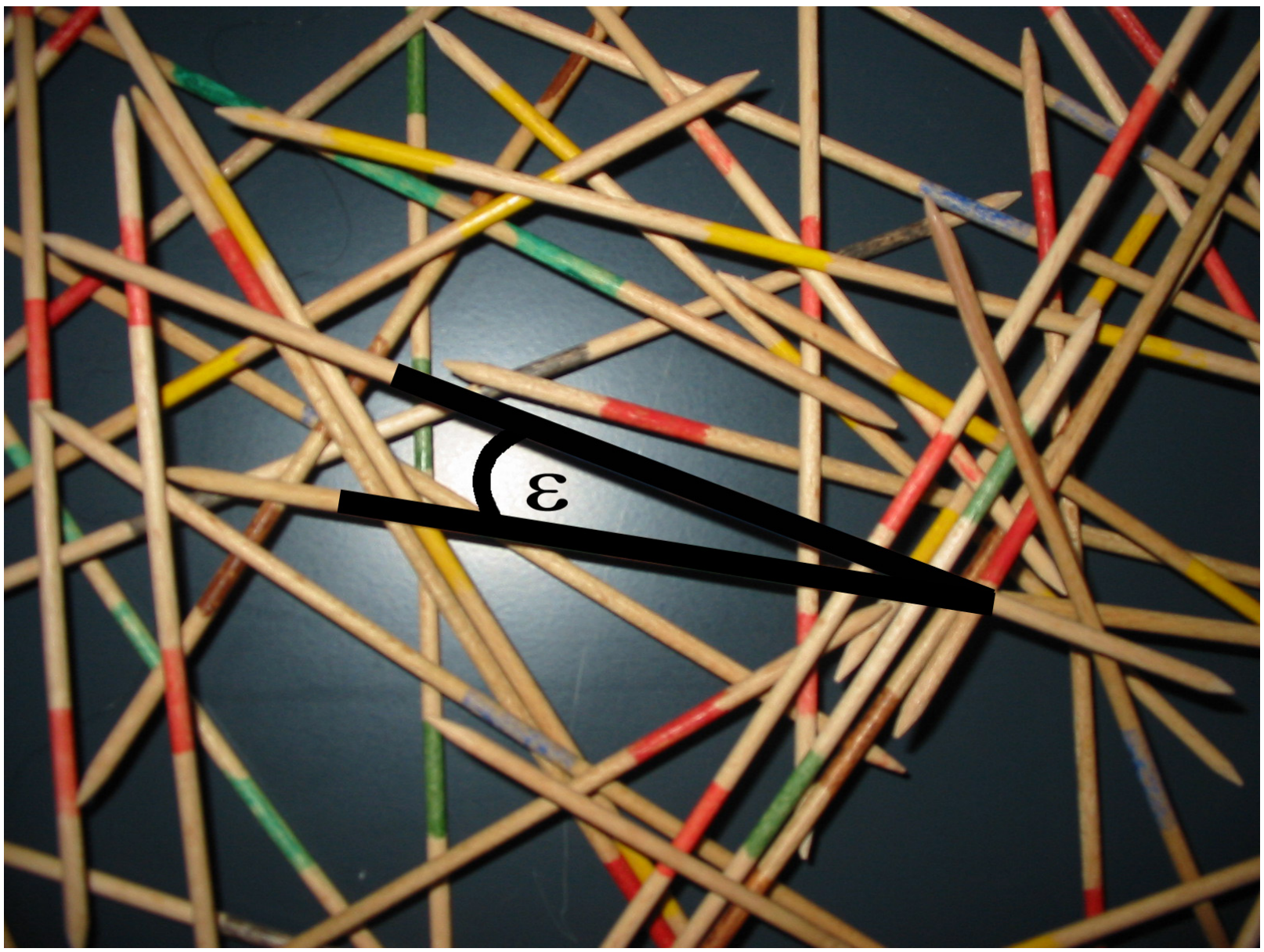

$$
P(\varepsilon, T) \sim \sin \varepsilon\left[T-2 T_{\text {enc }}(\varepsilon)\right]^{2}, T_{\text {enc }}=\frac{2}{\lambda} \ln \frac{c}{\epsilon}
$$




\section{Form factor: Off-diagonal contributions}

- leading-order loop contribution:

- action difference:

$$
\Delta S(\varepsilon) \simeq p^{2} \varepsilon^{2} /(2 m \lambda)
$$

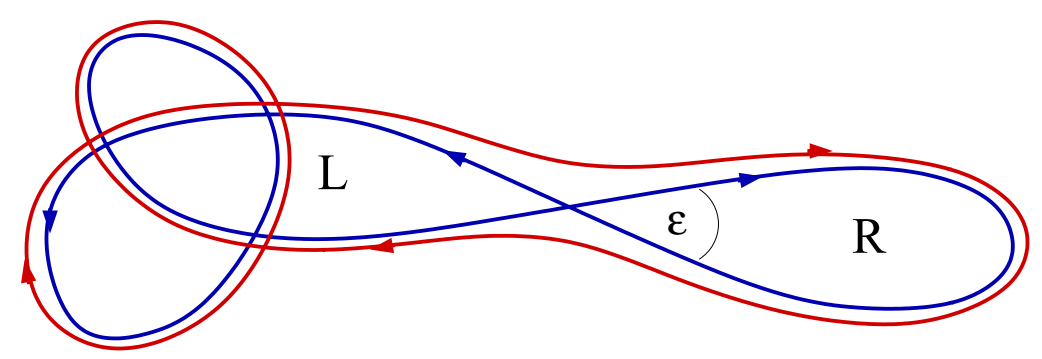

- contribution to form factor:

$$
K_{\text {off }}(\tau) \sim \operatorname{Re} \int_{0}^{\pi} \mathrm{d} \varepsilon \sum_{\gamma}\left|A_{\gamma}\right|^{2} e^{i \Delta S(\varepsilon) / \hbar} \delta\left(T-T_{\gamma}\right) P(\varepsilon, T)
$$

- density of crossings $P(\varepsilon, T)$ ?

$$
P(\varepsilon, T)=\frac{v^{2}}{2 \pi A} \sin \varepsilon\left[T-2 T_{\mathrm{enc}}(\varepsilon)\right]^{2}
$$

$$
\Longrightarrow \quad K_{\text {off }}(\tau) \approx-2 \tau^{2}
$$




\section{form factor: semiclassical evaluation}

$$
\begin{aligned}
K^{\mathrm{GOE}}(\tau) & =2 \tau-\tau \log (1+2 \tau) \\
& =2 \tau-2 \tau^{2}+\ldots \quad \text { for } \quad \tau=T / T_{\mathrm{H}}<1
\end{aligned}
$$

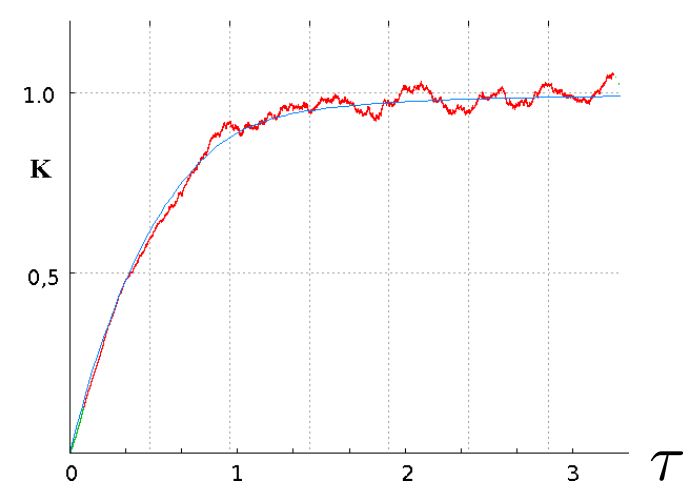

- phase space approaches:

D. Spehner (2003); M. Turek and KR (2003);

S Heusler, S Müller, P Braun, F Haake (2004)

- periodic orbit theory for the complete RMT form factor

Müller, Heusler, Altland, Braun, Haake (2007) 


\section{form factor: semiclassical evaluation}

$$
\begin{aligned}
K^{\mathrm{GOE}}(\tau) & =2 \tau-\tau \log (1+2 \tau) \\
& =2 \tau-2 \tau^{2}+\ldots \quad \text { for } \quad \tau=T / T_{\mathrm{H}}<1
\end{aligned}
$$

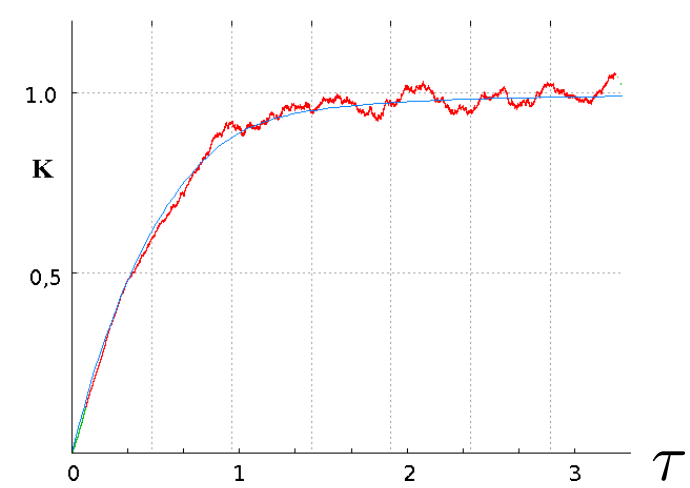

- phase space approaches:

D. Spehner (2003); M. Turek and KR (2003);

S Heusler, S Müller, P Braun, F Haake (2004)

- periodic orbit theory for the complete RMT form factor

Müller, Heusler, Altland, Braun, Haake (2007)

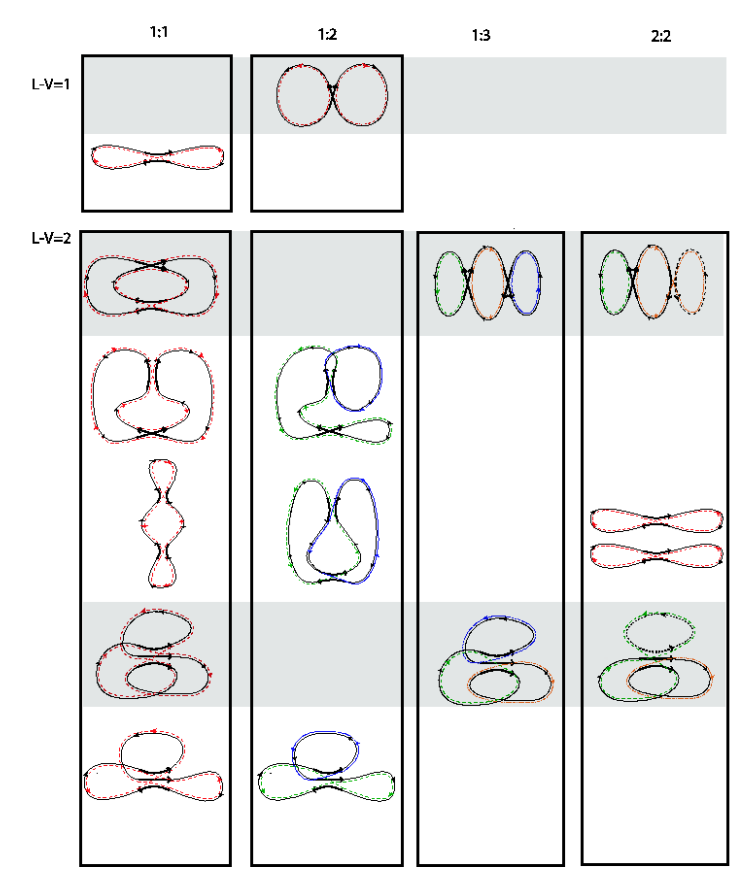




\section{form factor: semiclassical evaluation}

$$
\begin{aligned}
K^{\mathrm{GOE}}(\tau) & =2 \tau-\tau \log (1+2 \tau) \\
& =2 \tau-2 \tau^{2}+\ldots \quad \text { for } \quad \tau=T / T_{\mathrm{H}}<1
\end{aligned}
$$

- phase space approaches:

D. Spehner (2003); M. Turek and KR (2003);

S Heusler, S Müller, P Braun, F Haake (2004)

- periodic orbit theory for spectral statistics

$\rightarrow$ Sebastian Müller's lecture!

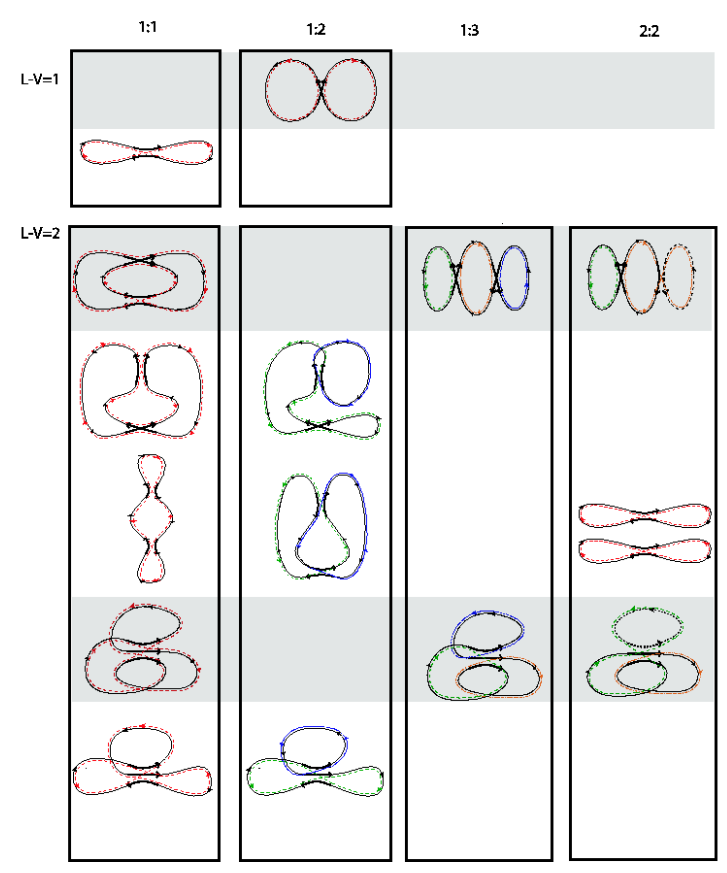




\section{form factor for the diffusive case}

- quantum (impurity) scattering takes role of encounter dynamics

- diagonal contributions sufficient

- relation between $K(t)$ and return probability $p(t)$ :

$$
K(t)=\frac{2}{(2 \pi \hbar)^{2}} \frac{\mathrm{d} \Omega}{\mathrm{d} E} t p(t)
$$

- diffusive case: $p(t) \sim(4 \pi D t)^{-N / 2}$

$$
K^{\mathrm{diff}}(t)=\frac{2 \Omega_{x}}{(2 \pi \hbar)^{2}(4 \pi D)^{N / 2}} t^{1-N / 2}
$$

Argaman, Imry, Smilansky (1993) 


\section{form factor for the diffusive case}

- quantum (impurity) scattering takes role of encounter dynamics

- diagonal contributions sufficient

- relation between $K(t)$ and return probability $p(t)$ :

$$
K(t)=\frac{2}{(2 \pi \hbar)^{2}} \frac{\mathrm{d} \Omega}{\mathrm{d} E} t p(t)
$$

- diffusive case: $p(t) \sim(4 \pi D t)^{-N / 2}$

$$
K^{\mathrm{diff}}(t)=\frac{2 \Omega_{x}}{(2 \pi \hbar)^{2}(4 \pi D)^{N / 2}} t^{1-N / 2}
$$

Argaman, Imry, Smilansky (1993)

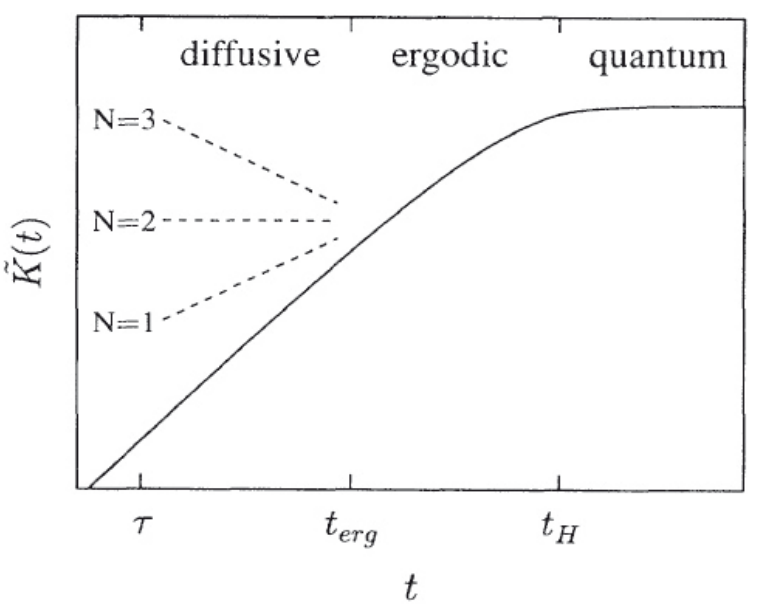




\section{Semiclassical $S$-matrix}




\section{$S$-matrix based observables}

- Wigner time delay:

$$
\tau_{\mathrm{W}}(E)=-\frac{i \hbar}{\mathrm{N}} \operatorname{Tr}\left[S^{\dagger}(E) \frac{\mathrm{d} S(E)}{\mathrm{d} E}\right]
$$




\section{$S$-matrix based observables}

- Wigner time delay:

$$
\tau_{\mathrm{W}}(E)=-\frac{i \hbar}{\mathrm{N}} \operatorname{Tr}\left[S^{\dagger}(E) \frac{\mathrm{d} S(E)}{\mathrm{d} E}\right]
$$

- mesoscopic quantum transport:

Landauer: "conductance is transmission": $G=\left(2 e^{2} / h\right) \operatorname{Tr}\left[t t^{\dagger}\right]$

- moments of transmission:

$$
M_{n}=\operatorname{Tr}\left\langle\left[t t^{\dagger}\right]^{n}\right\rangle
$$

$\rightarrow$ shot noise $\sim M_{1}-M_{2}$

$\rightarrow$ full counting statistics

- Andreev billiards 


\section{Semiclassical approach}

- Scattering matrix elements as sum over classical paths:

$$
S_{b a}(E) \sim \sum_{\gamma(a \rightarrow b)} A_{\gamma} e^{(i / \hbar) S_{\gamma}(E)}
$$

Miller (1972) ; Blümel and Smilansky (1988) 


\section{Semiclassical approach}

- Scattering matrix elements as sum over classical paths:

$$
S_{b a}(E) \sim \sum_{\gamma(a \rightarrow b)} A_{\gamma} e^{(i / \hbar) S_{\gamma}(E)}
$$

Miller (1972) ; Blümel and Smilansky (1988)

- Semiclassical treatment of correlation functions:

$$
C(\epsilon, n)=\operatorname{Tr}\left\langle\left[S^{\dagger}(E-\epsilon) S(E+\epsilon)\right]^{n}\right\rangle
$$

trajectory sets with encounters contributing e.g. to $n=3$ :

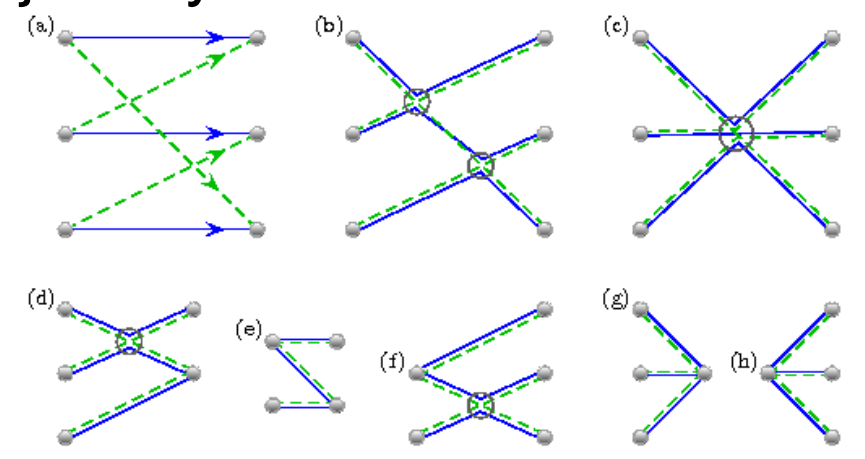

treatment to leading order in $1 / N$ :

Berkolaiko, Harrison, Novaes, JPA (2008)

Ehrenfest time dependence:

Waltner, Kuipers, Richter, arXive:1007:1595 


\section{Andreev billiards: semiclassics beyond RMT}




\section{Andreev reflection}

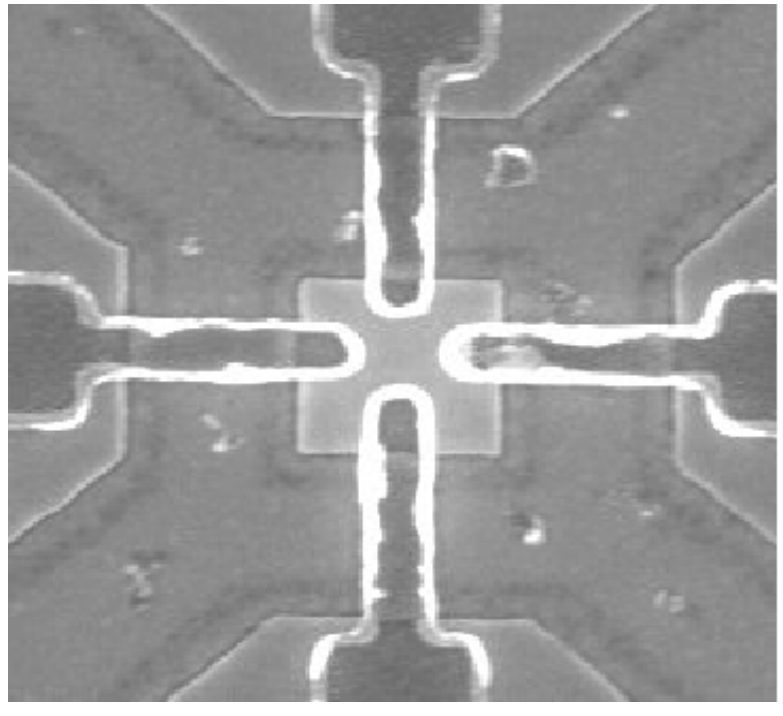

A. Philip, Groningen
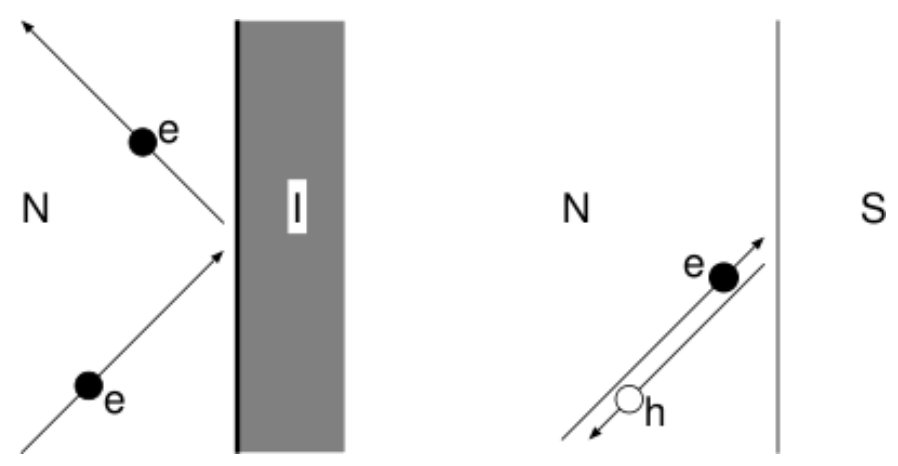

retro-reflection at normal-superconductor-interface 


\section{Andreev Billiards}

Andreev (retro-) reflection at superconductor-billiard interface

$\Rightarrow$ periodic orbits

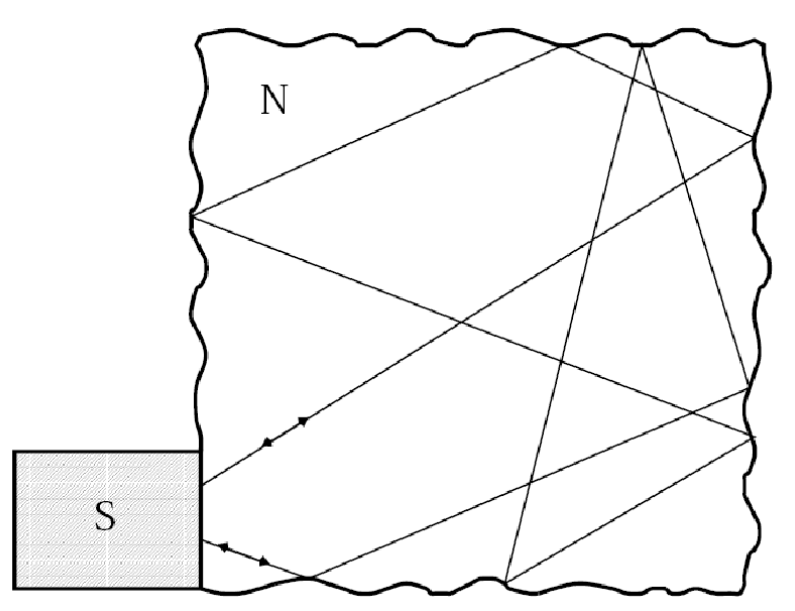

- 'Bohr-Sommerfeld' expression for DoS in billiard: $S_{\gamma}=(n+1 / 2) h$

$$
\Rightarrow \quad d(E) \sim \int_{0}^{\infty} \mathrm{d} L P(L) L \sum_{n} \delta\left(\frac{E L}{\hbar v_{F}}-\left(n+\frac{1}{2}\right) \pi\right)
$$

Melsen, Brouwer, Frahm, Beenakker (1996) ; Lodder \& Nazarov (1998)

- probe $P(L)$, i.e classical dynamics, through proximity effect ! 


\section{Proximity effect on density of states}

$$
d(E) \sim \int_{0}^{\infty} \mathrm{d} L P(L) L \sum_{n} \delta\left(\frac{E L}{\hbar v_{F}}-\left(n+\frac{1}{2}\right) \pi\right)
$$

chaotic case: $P(L) \sim e^{-L / L_{D}} \quad$ regular case: $P(L) \sim L^{-\alpha}$

square billiard (rough walls)

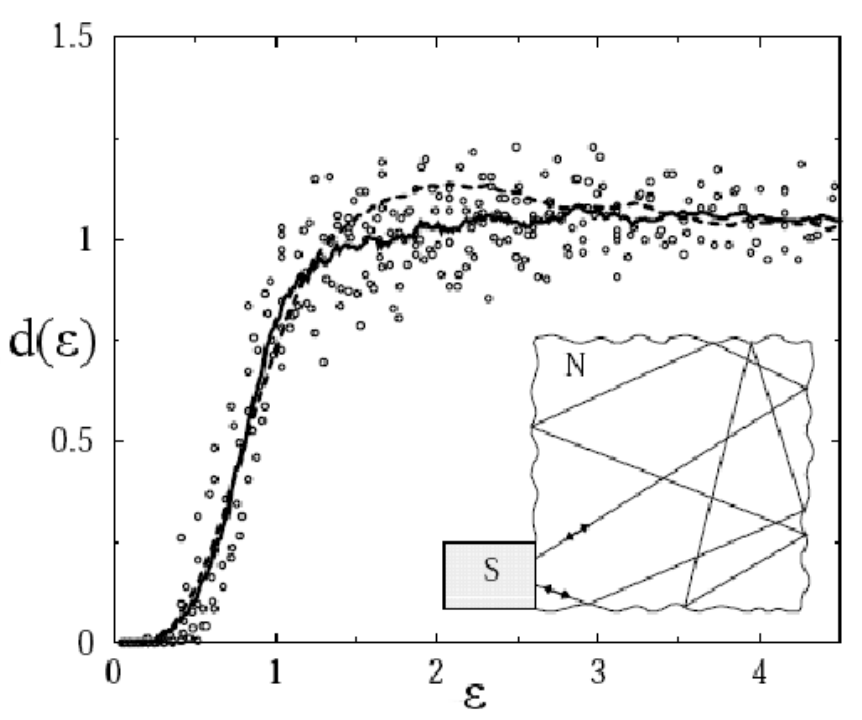

gap in DoS square billiard

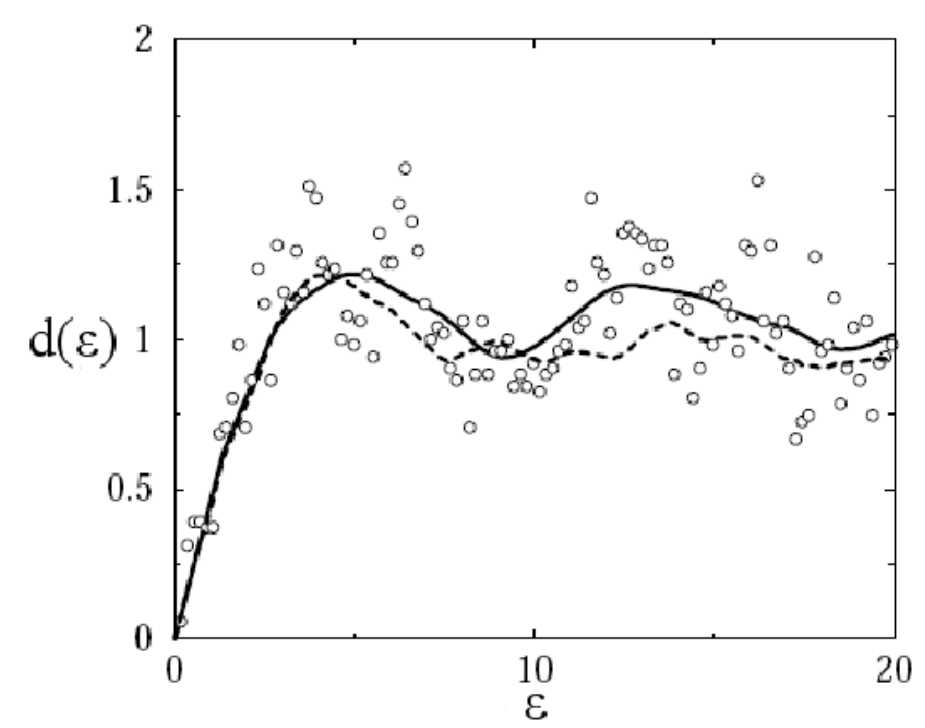

linear suppression of DoS 


\section{Excitation gap in chaotic case}

\section{Random Matrix Theory:}

hard gap

at Thouless energy: $E_{T}=\frac{\hbar}{2 \tau_{D}}$

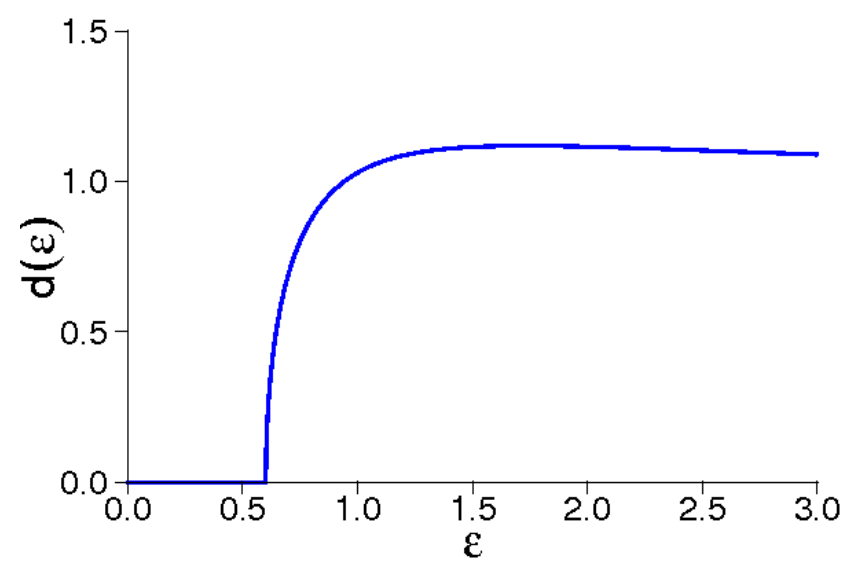

$\epsilon=E / E_{\mathrm{T}}$

Melsen, Brouwer, Frahm

\& Beenakker (1996)

\section{'Bohr-Sommerfeld':}

no gap

( diagonal approximation)

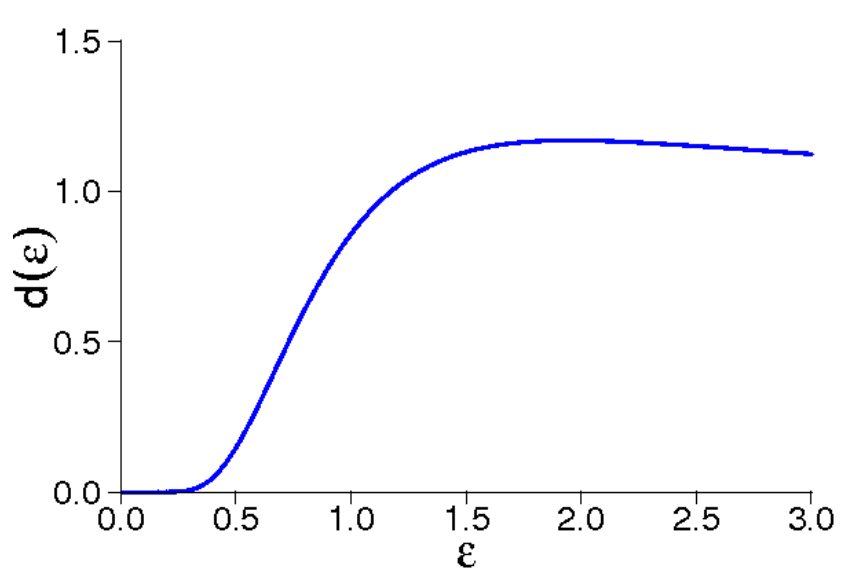

Schomerus \& Beenakker (1999);

Ihra, Leadbeater, Vega, KR \& (2001) 


\section{Excitation gap in chaotic case}

\section{Random Matrix Theory:}

hard gap

at Thouless energy: $E_{T}=\frac{\hbar}{2 \tau_{D}}$

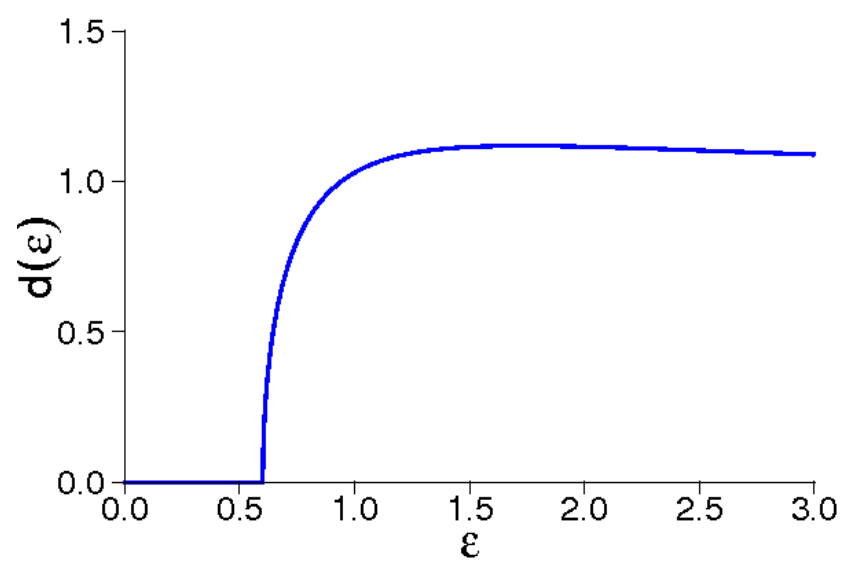

$\epsilon=E / E_{\mathrm{T}}$

Melsen, Brouwer, Frahm

\& Beenakker (1996)

\section{'Bohr-Sommerfeld':}

no gap

( diagonal approximation)

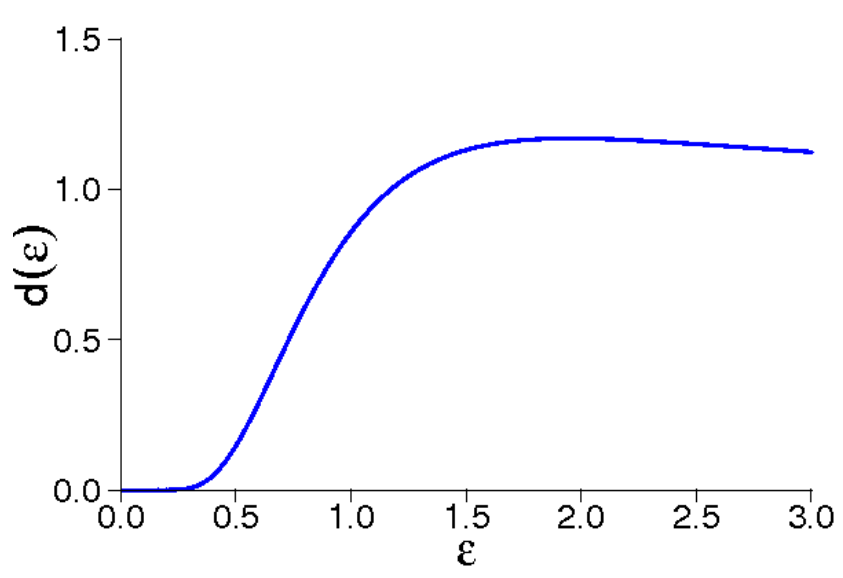

Schomerus \& Beenakker (1999); Ihra, Leadbeater, Vega, KR \& (2001)

way out: Ehrenfest time dependence Lodder \& Nazarov (1998) 


\section{Ehrenfest time effects}

- Ehrenfest time $\tau_{\mathrm{E}}$ : $\quad$ Chirikov et al. (1981)

separates wavepacket evolution following classical dynamics from time scales dominated by (random) wave interference
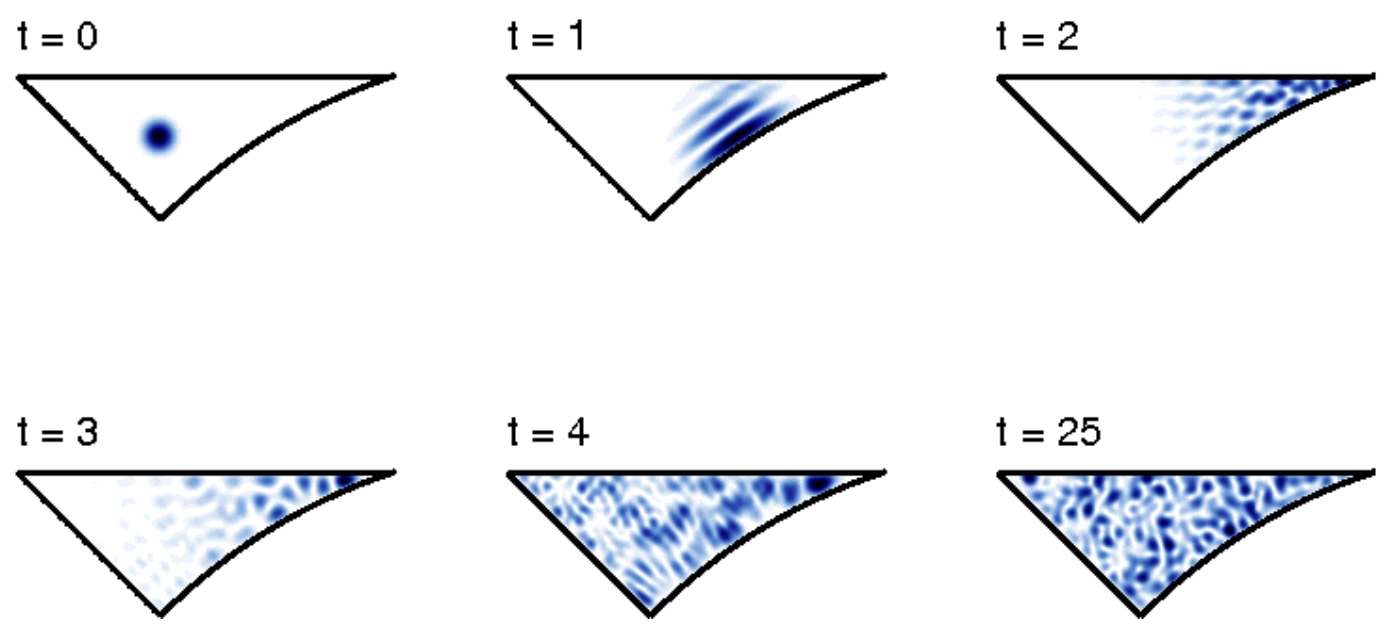

- $\mathcal{L}=\lambda_{\mathrm{dB}} \exp \left(\lambda \tau_{E}\right) \quad ; \quad \tau_{E} \sim|\ln \hbar|$

- short time correction to quantum universality 


\section{Excitation gap in chaotic case}

Random Matrix Theory:

hard gap

at Thouless energy: $E_{T}=\frac{\hbar}{2 \tau_{D}}$

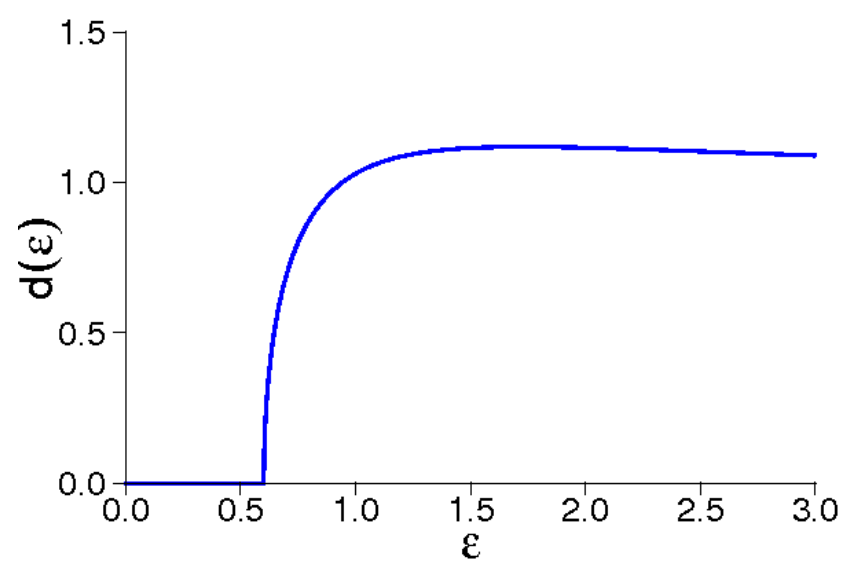

$\epsilon=E / E_{\mathrm{T}}$
'Bohr-Sommerfeld':

no gap

( diagonal approximation)

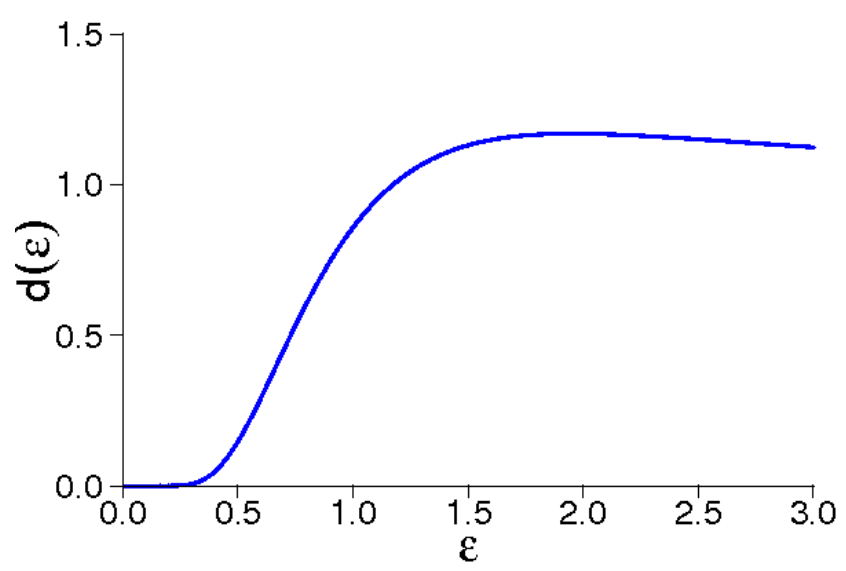

universal regime

$\tau=\tau_{E} / \tau_{D} \rightarrow 0$ "classical" limit

$\tau=\tau_{E} / \tau_{D} \rightarrow \infty$ 


\section{Excitation gap in chaotic case}

\section{Random Matrix Theory:}

hard gap

at Thouless energy: $E_{T}=\frac{\hbar}{2 \tau_{D}}$

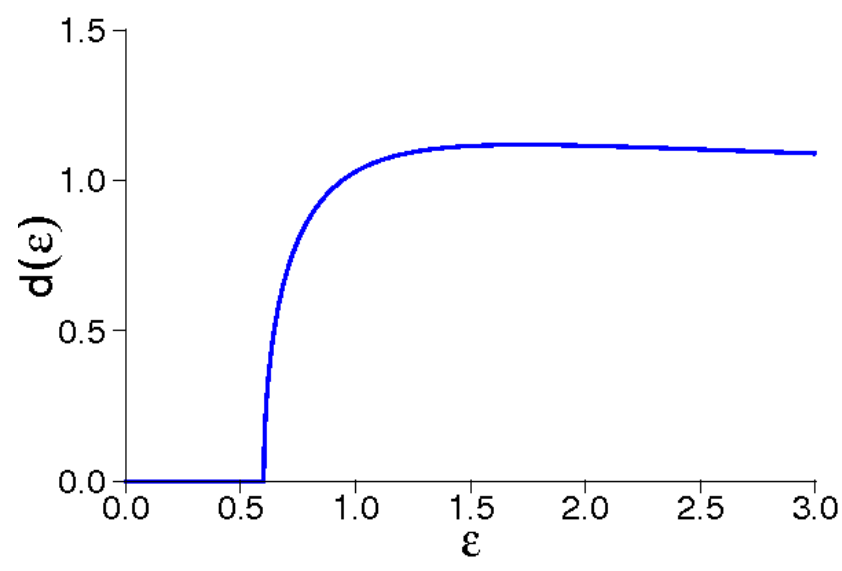

$\epsilon=E / E_{\mathrm{T}}$

universal regime

$$
\tau=\tau_{E} / \tau_{D} \rightarrow 0
$$

'Bohr-Sommerfeld':

no gap

( diagonal approximation)

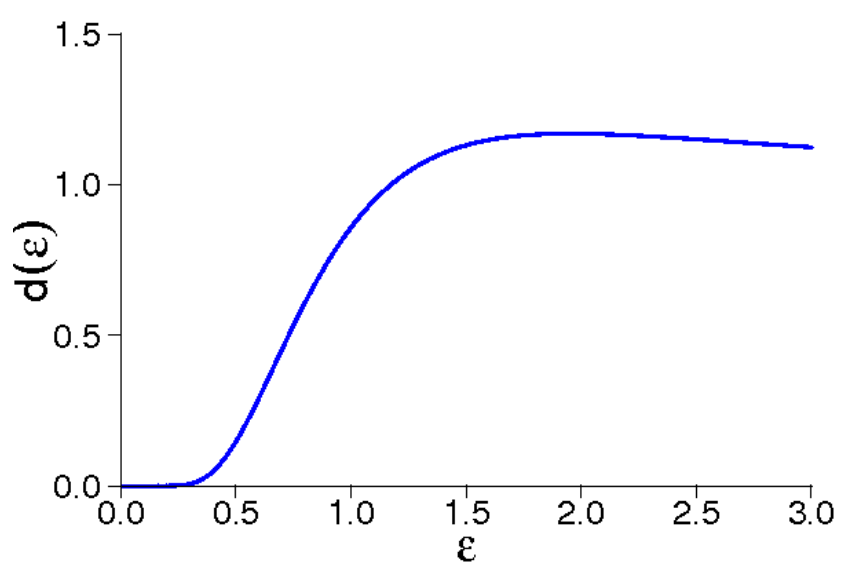

- semiclassical mechanism for hard RMT gap ?

- crossover: universal $\longrightarrow$ "classical" limit ? 


\section{Scattering approach to DoS}

\section{Scattering matrix:}

$$
\begin{aligned}
\mathbf{S}= & \mathbf{S}_{\mathrm{N}} \mathbf{S}_{\mathrm{A}} \\
= & \left.\begin{array}{cc}
S(E) & 0 \\
0 & S^{*}(-E)
\end{array}\right)\left(\begin{array}{cc}
0 & -i \\
-i & 0
\end{array}\right)
\end{aligned}
$$

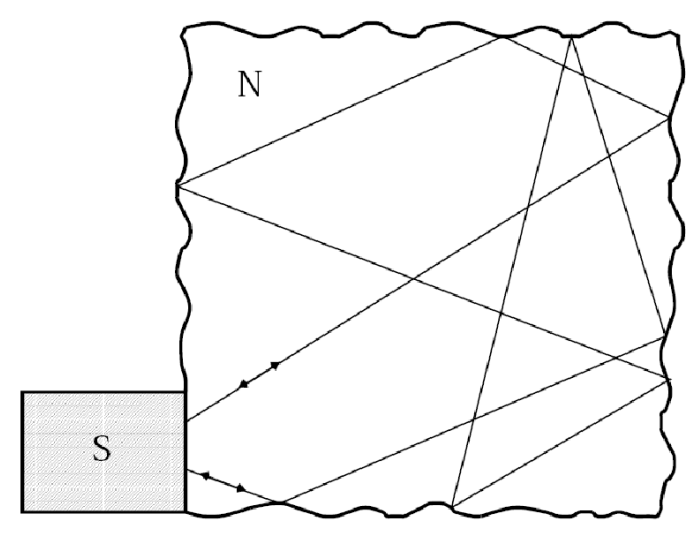

(e) part (h) part Andreev reflect. 


\section{Scattering approach to DoS}

\section{Scattering matrix:}

$$
\begin{aligned}
\mathbf{S} & =\mathbf{S}_{\mathrm{N}} \mathbf{S}_{\mathrm{A}} \\
& =\left(\begin{array}{cc}
S(E) & 0 \\
0 & S^{*}(-E)
\end{array}\right)\left(\begin{array}{cc}
0 & -i \\
-i & 0
\end{array}\right)
\end{aligned}
$$

$\uparrow$

$\uparrow$

$\uparrow$

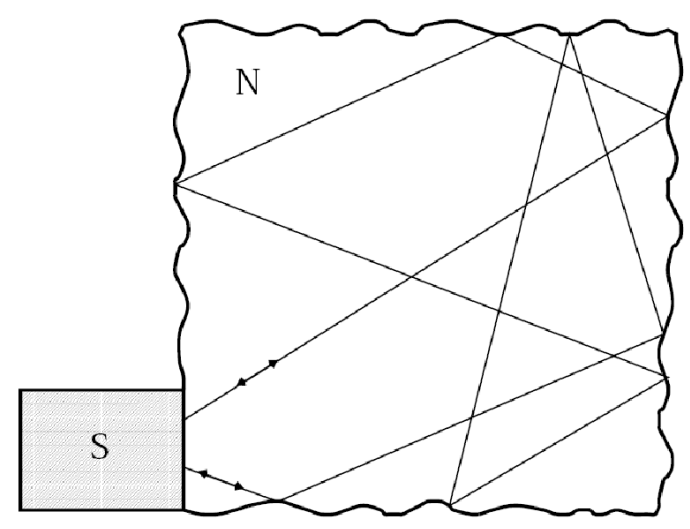

(e) part (h) part Andreev reflect.

Density of states in terms of scattering matrix:

$$
d(\epsilon) \sim 1+\frac{2}{M} \sum_{n=1}^{\infty} \frac{(-1)^{n}}{n} \frac{\partial}{\partial \epsilon} \operatorname{Im}\{\underbrace{\operatorname{Tr}\left[S^{\dagger}\left(E_{F}-\frac{\epsilon \hbar}{2 \tau_{D}}\right) S\left(E_{F}+\frac{\epsilon \hbar}{2 \tau_{D}}\right)\right]^{n}}_{\equiv C(\epsilon, n)}\}
$$




\section{Semiclassical approach}

- Scattering matrix elements as sum over classical paths:

$$
S_{b a}(E) \sim \sum_{\gamma(a \rightarrow b)} A_{\gamma} e^{(i / \hbar) S_{\gamma}(E)}
$$




\section{Semiclassical approach}

- Scattering matrix elements as sum over classical paths:

$$
S_{b a}(E) \sim \sum_{\gamma(a \rightarrow b)} A_{\gamma} e^{(i / \hbar) S_{\gamma}(E)}
$$

- Correlation functions $C(\epsilon, n)=\operatorname{Tr}\left[S^{\dagger}(-\epsilon) S(\epsilon)\right]^{n}$ in terms of sums over $2 n$ paths

- trajectory structures of interfering paths $(n=3)$ :
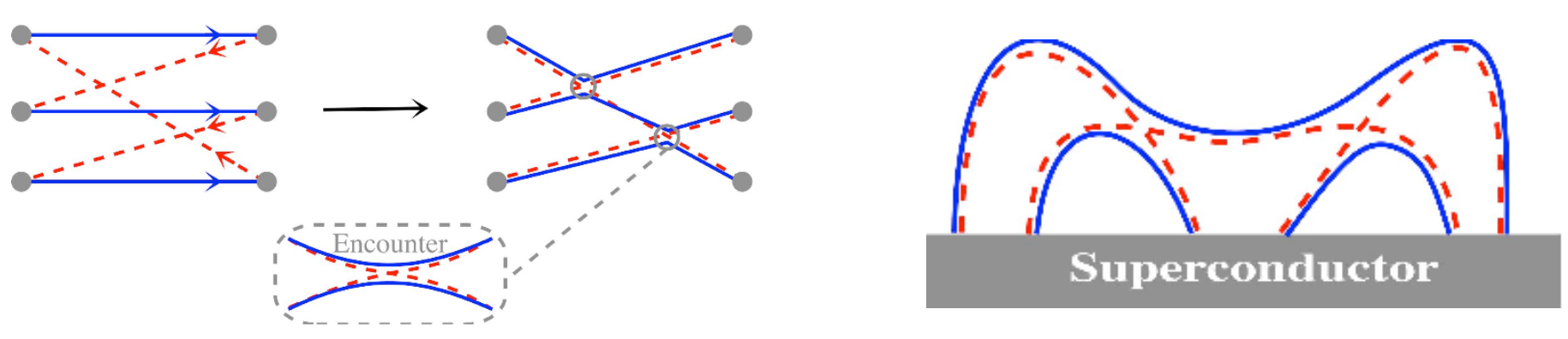


\section{Evaluation of diagrams}
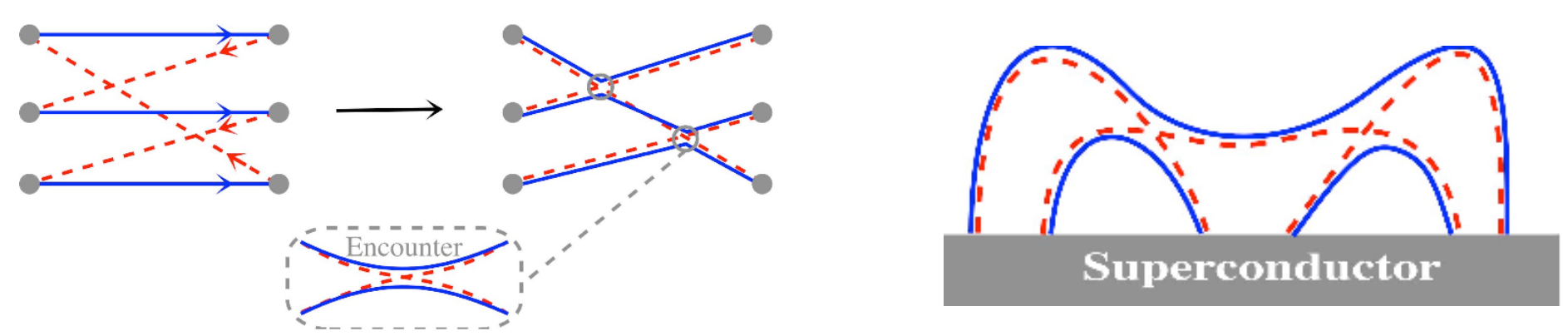

- apply diagrammatic rules: (Heusler et al. (2006))

- extend work on full counting statistics (Berkolaiko, Harrison \& Novaes (2008))

$\rightarrow$ generating function of correlators (which includes all diagrams):

$$
G(\epsilon, r)=\sum_{n=1}^{\infty} r^{n-1} C(\epsilon, n)
$$




\section{Evaluation of diagrams}

- DoS in terms of the generating function:

$$
\begin{aligned}
H(\epsilon, r) & =\frac{1}{r} \frac{\partial}{\partial \epsilon} \int G(\epsilon, r) \mathrm{d} r=\sum_{n=1}^{\infty} \frac{r^{n-1}}{n} \frac{\partial C(\epsilon, n)}{\partial \epsilon} \\
d(\epsilon) & =1-2 \operatorname{Im} H(\epsilon,-1)
\end{aligned}
$$

- DoS given by solutions of a cubic equation 


\section{Evaluation of diagrams}

- DoS in terms of the generating function:

$$
\begin{aligned}
H(\epsilon, r) & =\frac{1}{r} \frac{\partial}{\partial \epsilon} \int G(\epsilon, r) \mathrm{d} r=\sum_{n=1}^{\infty} \frac{r^{n-1}}{n} \frac{\partial C(\epsilon, n)}{\partial \epsilon} \\
d(\epsilon) & =1-2 \operatorname{Im} H(\epsilon,-1)
\end{aligned}
$$

- DoS given by solutions of a cubic equation

This yields the RMT result

Kuipers, Waltner, Petitjean, Berkolaiko, KR,

Phys. Rev. Lett. (2010)

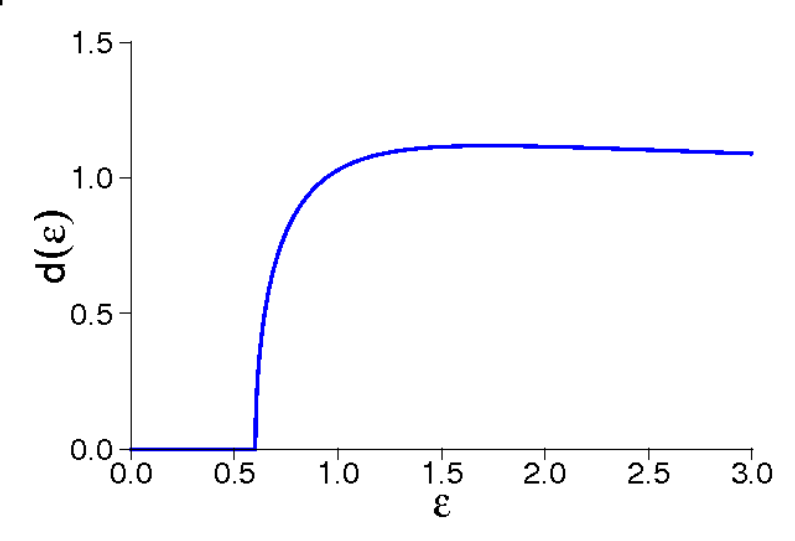




\section{Finite Ehrenfest time}

\section{RMT limit:}

$$
\tau=\tau_{E} / \tau_{D} \rightarrow 0
$$

hard gap

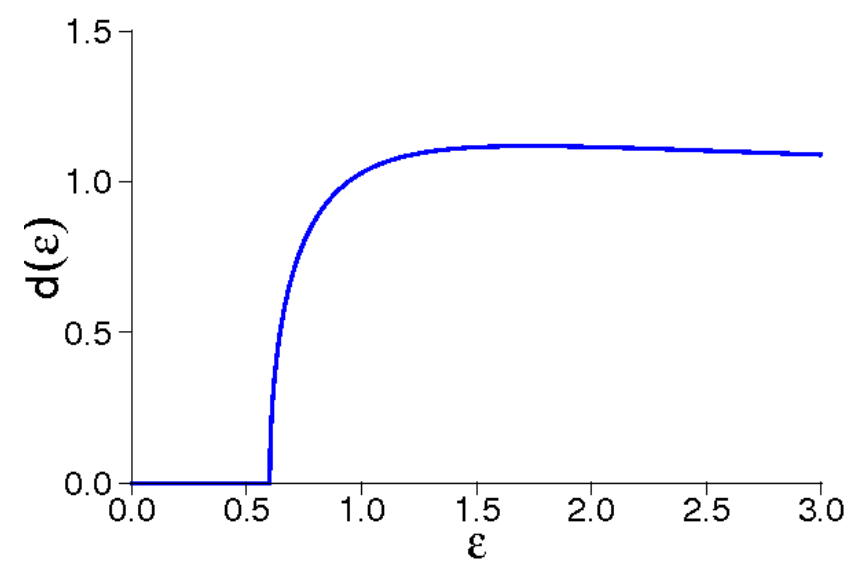

"classical" limit:

$\tau=\tau_{E} / \tau_{D} \rightarrow \infty$

no gap

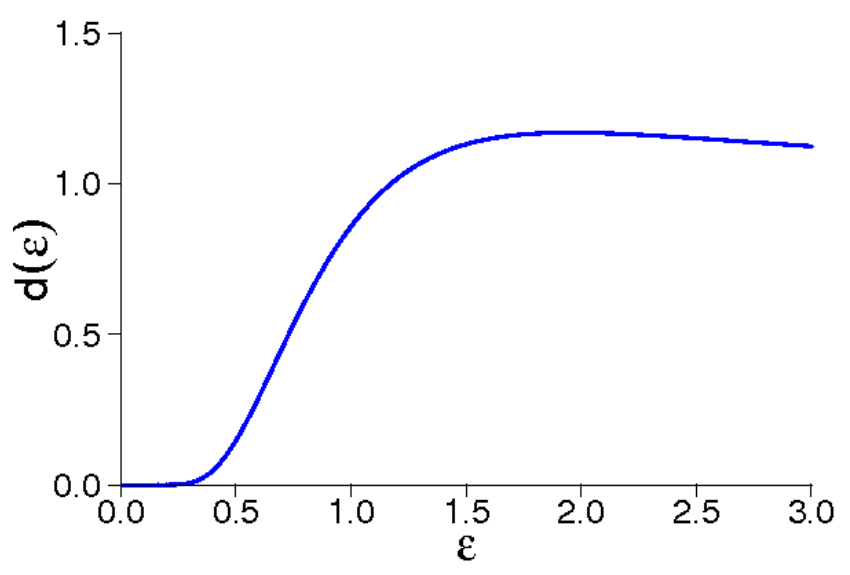

crossover: universal RMT $\rightarrow$ "classical" limit ?

Ehrenfest time dependence of the gap width? 


\section{Finite Ehrenfest time}

each encounter lasts an Ehrenfest time $\tau_{E}$ :

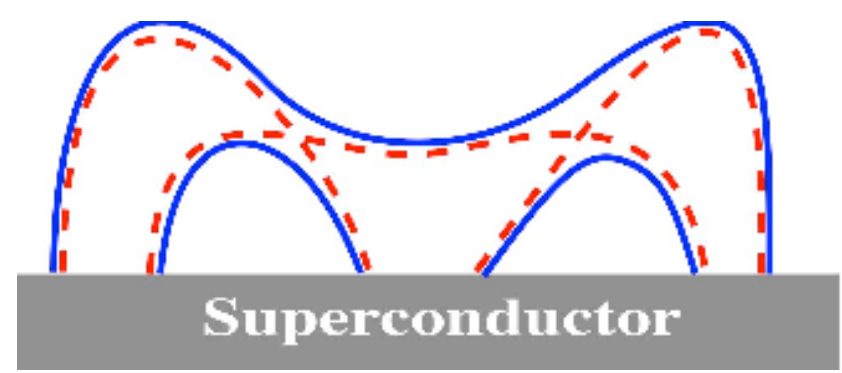

increase of $\tau_{E}$ :

forming diagrams with encounters is less likely

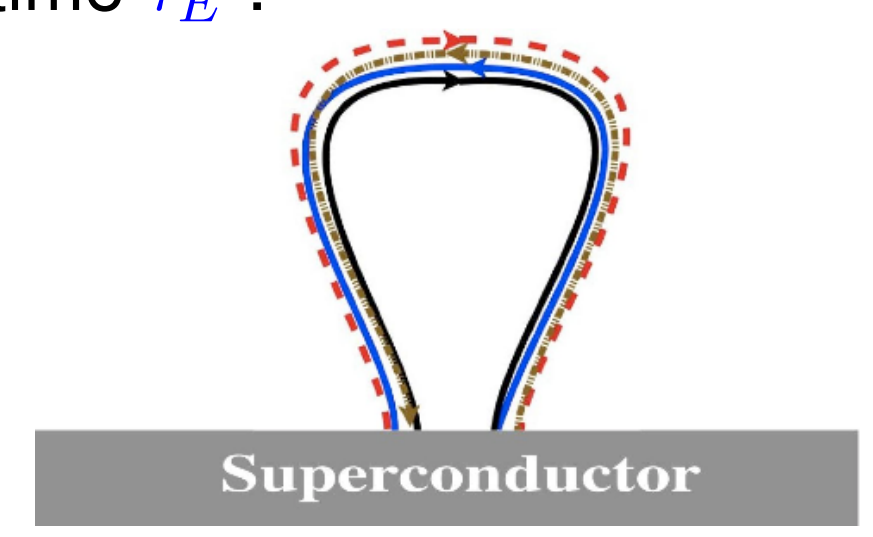

increase of $\tau_{E}$ : possiblity increases for diagrams with correlated trajectories along their whole length

$$
C(\epsilon, \tau, n)=C(\epsilon, n) e^{-\tau(1-i n \epsilon)}+\frac{1-e^{-\tau(1-i n \epsilon)}}{1-i n \epsilon} \quad \tau=\tau_{E} / \tau_{D}
$$

see also: Whitney \& Jacquod (2006); Brouwer \& Rahav (2006) for $n \leq 3$ 


\section{Finite Ehrenfest time}

- intermediate Ehrenfest times: $\tau=\tau_{E} / \tau_{D}=2$

\section{Oscillations}

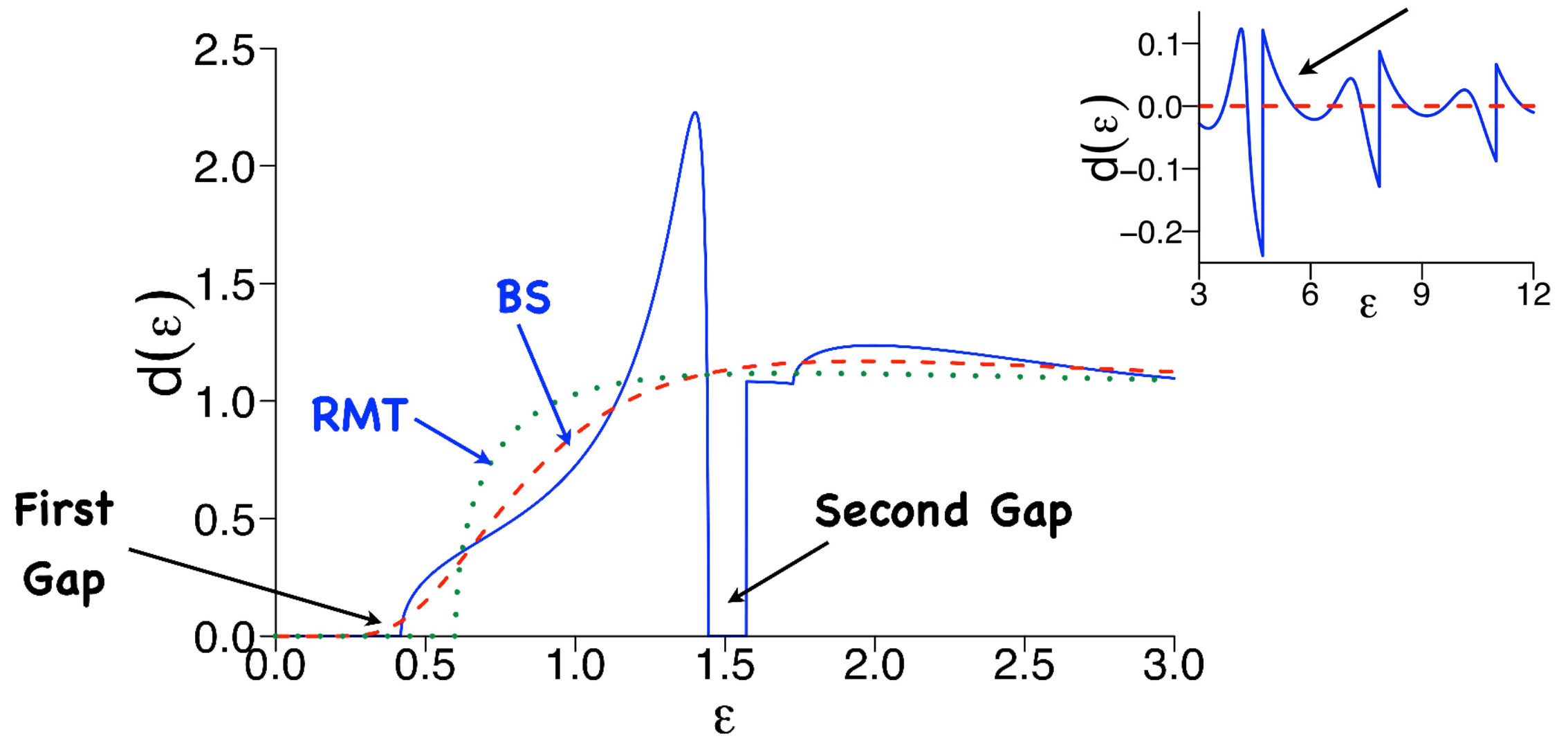




\section{Finite Ehrenfest time}

- intermediate Ehrenfest times: $\tau=\tau_{E} / \tau_{D}=2$

\section{Oscillations}

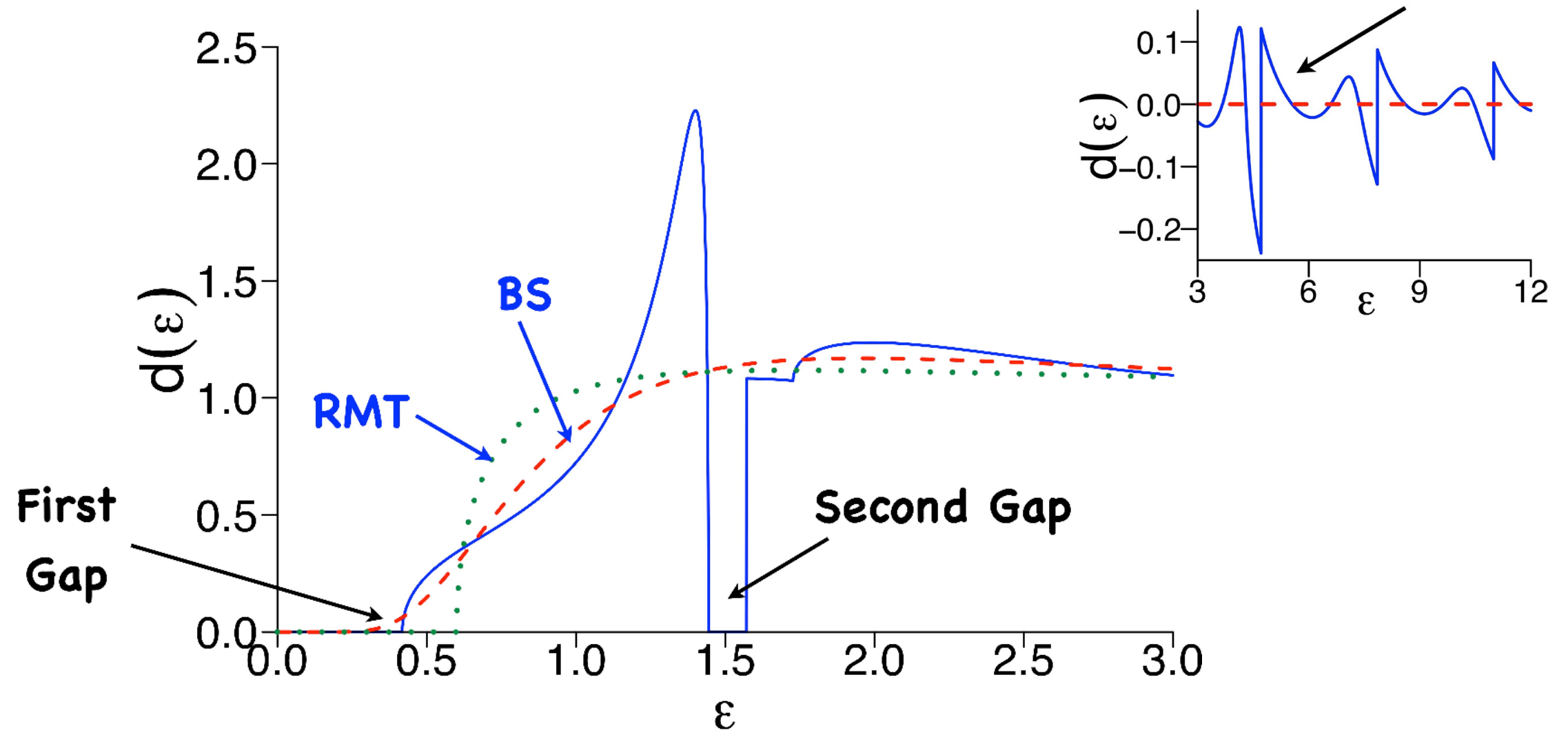

- second gap at 'Ehrenfest energy' $E_{\mathrm{E}}=\pi \hbar / 2 \tau_{E}$ !

Kuipers, Waltner, Petitjean, Berkolaiko, KR, Phys. Rev. Lett. (2010)

Kuipers, Engl, Berkolaiko, Petitjean, Waltner, KR, arXiv:1004.1327 


\section{Summary and outlook}

Advanced semiclassics can:

- explain universality of chaotic quantum systems

- go beyond random matrix theory 


\section{Summary and outlook}

\section{Advanced semiclassics can:}

- explain universality of chaotic quantum systems

- go beyond random matrix theory

- tackle observables in the mesoscopic regime:

- superconducting-normal conducting hybrids

- quantum decay, time delay

- fidelity

- quantum transport (weak and strong localization)

- treat multi-component wave (Green) function structures:

- spin-orbit interaction in low-dimensional semiconductors

- pseudospin- and valley-degrees of freedom in graphene

- Luttinger hamiltonian for holes 


\section{Thanks to:}

- Jack Kuipers, Cyril Petitjean, Daniel Waltner, Thomas Engl, Gregory Berkolaiko

- Deutsche Forschungsgemeinschaft (FOR 760, GRK 638), Alexander von Humboldt Foundation 